Prepared For SUBMission to JCAP

\title{
Uniqueness of the gauge invariant action for cosmological perturbations
}

\author{
Tomislav Prokopec and Jan Weenink \\ Institute for Theoretical Physics and Spinoza Institute, \\ Utrecht University, Leuvenlaan 4, 3585 CE Utrecht, The Netherlands \\ E-mail: t.prokopec@uu.nl,j.g.weenink@uu.nl
}

\begin{abstract}
In second order perturbation theory different definitions are known of gauge invariant perturbations in single field inflationary models. Consequently the corresponding gauge invariant cubic actions do not have the same form. Here we show that the cubic action for one choice of gauge invariant variables is unique in the following sense: the action for any other, non-linearly related variable can be brought to the same bulk action, plus additional boundary terms. These boundary terms correspond to the choice of hypersurface and generate extra, disconnected contributions to the bispectrum. We also discuss uniqueness of the action with respect to conformal frames. When expressed in terms of the gauge invariant curvature perturbation on uniform field hypersurfaces the action for cosmological perturbations has a unique form, independent of the original Einstein or Jordan frame. Crucial is that the gauge invariant comoving curvature perturbation is frame independent, which makes it extremely helpful in showing the quantum equivalence of the two frames, and therefore in calculating quantum effects in nonminimally coupled theories such as Higss inflation.
\end{abstract}




\section{Contents}

1 Introduction 1

2 Action and perturbations 2

3 Gauge dependence and gauge invariant perturbations 4

3.1 Gauge transformations of metric and field fluctuations 4

3.2 Constructing gauge invariant variables 5

4 The gauge invariant action for cosmological perturbations $\quad 7$

$\begin{array}{lll}4.1 & \text { Gauge invariance at zeroth order } & 7\end{array}$

4.2 Gauge invariance of quadratic action 8

4.3 Gauge invariance of cubic action 8

4.3.1 Manifest gauge invariance: cubic action for $W_{\varphi} \quad 9$

4.3.2 Manifest gauge invariance: cubic action for $W_{\zeta} \quad 11$

5 Uniqueness of gauge invariant action $\quad 12$

5.1 Non-linear transformations 13

$\begin{array}{lll}5.2 & \text { Practical example: different gauge invariant variables } & 15\end{array}$

$\begin{array}{lll}5.3 & \text { Boundary terms, hypersurfaces and observations } & 16\end{array}$

6 Frame independent cosmological perturbations $\quad 18$

6.1 Einstein frame and Jordan frame 18

6.2 Frame independence of second order action 20

6.3 Frame independence of cubic action 21

7 Summary and conclusion $\quad 24$

8 Acknowledgements 25

\section{Introduction}

The detection of non-Gaussianities in the Cosmic Microwave Background radiation (CMB) would provide a wealth of information. From the point of view of inflationary physics, it would constrain the correlation functions of primordial fluctuations. Since these correlation functions can be explicitly found for one of the many inflationary theories, non-Gaussianity provides a powerful tool to constrain the parameters in these theories and discriminate between models in the inflationary zoo.

One of the simplest inflationary models is that of a single scalar field in a slow-roll scenario. In his seminal work [1] Maldacena found the third order action for inflationary perturbations and showed that non-Gaussianities are too small to be observed. This has been extended to more general scalar theories in [2] and [3].

Importantly, the cubic action and 3-point functions have been derived for $\zeta$, the curvature perturbation. It is well-known that $\zeta$ itself is a gauge dependent quantity, in the sense that it is not invariant under reparametrizations of coordinates. Only a gauge invariant perturbation 
can be called physical. On the other hand one can work with gauge fixed quantities, although one must be careful with respect to gauge artifacts.

In this work we set out to find the cubic action for gauge invariant cosmological perturbations. We show how to do this for different gauge invariant variables at second order and that the gauge invariant actions reduce to the ones in [1] in the spatially flat or uniform field gauge. In general, the gauge invariant cubic actions for different variables are different, but we show that the bulk part of the gauge invariant action coincides for different variables. In that sense the evolution of non-Gaussianity is unique. The difference between the actions lies in boundary terms, which are associated with the choice of hypersurface. They generate additional, disconnected parts of the bispectrum.

In the second part of this work we discuss uniqueness from the point of view of conformally related frames. As is well known, nonminimally coupled actions, or Jordan frame actions, can be written into a minimally coupled form, the Einstein frame, by field dependent redefinitions of the metric and scalar field. Thus in principle nontrivial Jordan frame results can be obtained from well-known Einstein frame results by redefining fields, which makes the frame transformation a very powerful tool. The situation becomes complicated at the level of perturbations. The problem is that the perturbations of the metric and scalar field in the Einstein frame are not equal to those in the Jordan frame, precisely due to the field dependent field redefinition. The situation is very similar to the gauge problem for perturbations.

In this work we point out that it is possible to construct frame independent cosmological perturbations, which are very useful to relate Einstein frame results to Jordan frame results. Here is where the second aspect of uniqueness comes in: the cubic action for cosmological perturbations takes a unique form, independent of the frame, provided one makes use of precisely that variable that is the same in either frame. As it turns out, this variable coincides with the gauge invariant comoving curvature perturbation.

A short outlook: in Sec. 2 we define the single scalar field action and its perturbations, in Sec. 3 we construct different gauge invariant variables at second order and in Sec. 4 we construct the gauge invariant action for cosmological perturbations and discuss uniqueness. In the second part, Sec. 6 we discuss different frames and perturbations in those frames, and show in what aspect the action is unique.

\section{Action and perturbations}

The action under consideration is the Einstein-Hilbert action for General Relativity plus a minimally coupled scalar field

$$
S=\int d^{4} x \sqrt{-g}\left\{\frac{1}{2} R-\frac{1}{2} g^{\mu \nu} \partial_{\mu} \Phi \partial_{\nu} \Phi-V(\Phi)\right\} .
$$

Here we use natural units in which $8 \pi G=1, c=1=\hbar$. This so-called Einstein frame action is manifestly covariant, that is, it is invariant under spacetime coordinate reparametrizations. It is possible to study the background field equations for (2.1). Taking as the background spacetime the homogeneous and isotropic FLRW metric leads for example to the Friedmann equations in presence of a time dependent scalar field, which give rise to inflationary solutions in certain regimes.

It is also possible to study perturbations of the metric and the scalar field in the action (2.1). A perturbation of a quantity is defined as the difference between the quantity in the physical spacetime and the quantity in the background spacetime. In order to compare these quantities 
one has to choose a mapping between the physical and background spacetimes. The gauge freedom in General Relativity is the freedom in choosing a mapping. As a consequence the perturbations themselves depend on the choice of mapping. They are in other words gauge dependent.

Gauge dependence is in principle problematic, since physical results should not depend on reparametrization of coordinates. However, we know for a fact that the original, unperturbed action (2.1) is explicitly covariant. Therefore, it may be possible to write the perturbed action in a manifestly covariant way. Indeed, this can be achieved using gauge invariant cosmological perturbations [4]. Gauge invariance here is synonymous to covariance or diffeomorphism invariance.

A convenient method to deal with the gauge dependence in the action is to use the ADM formalism [5] with line element

$$
d s^{2}=-N^{2} d t^{2}+g_{i j}\left(d x^{i}+N^{i} d t\right)\left(d x^{j}+N^{j} d t\right) .
$$

Geometrically, spacetime has been sliced up in spatial hypersurfaces whose geometry is described by the spatial metric $g_{i j}$. The slicing and threading of spacetime is described by the lapse function $N$ and shift functions $N_{i}$. The action (2.1) with the ADM metric (2.2) becomes

$$
S=\frac{1}{2} \int d^{3} x d t N \sqrt{g}\left\{R^{(3)}+N^{-2}\left(E^{i j} E_{i j}-E^{2}\right)+N^{-2}\left(\partial_{0} \Phi-N^{i} \partial_{i} \Phi\right)^{2}-g^{i j} \partial_{i} \Phi \partial_{j} \Phi-2 V(\Phi)\right\},
$$

where

$$
\begin{aligned}
E_{i j} & =\frac{1}{2}\left(\partial_{0} g_{i j}-\nabla_{i} N_{j}-\nabla_{j} N_{i}\right) \\
E & =g^{i j} E_{i j},
\end{aligned}
$$

and $R^{(3)}$ is the spatial scalar curvature computed from (spatial derivatives of) $g_{i j}$ alone. From Eq. (2.3) it is clear that $N$ and $N_{i}$ are non-dynamical fields, and moreover they are gauge dependent. In fact, in a Hamiltonian formulation they appear as Lagrange multipliers. Solving for these fields in the action (2.3) removes the unphysical degrees of freedom. In the end the only dynamical perturbations out of the 7 degrees of freedom in $g_{i j}$ and $\Phi$ are one scalar degree of freedom and a graviton. The remaining 4 degrees of freedom are nondynamical and are actually the solutions of the constraint equations [6]. The dynamical degrees of freedom are indeed gauge invariant.

The perturbed action is calculated by inserting

$$
\begin{aligned}
\Phi & =\phi(t)+\varphi \\
g_{i j} & =a(t)^{2}\left(\delta_{i j} e^{2 \zeta}+\frac{1}{a^{2}} \partial_{i} \partial_{j} \tilde{h}+\frac{1}{a} \partial_{(i} h_{j)}^{T}+h_{i j}^{T T}\right) \\
N & =\bar{N}(t)(1+n) \\
N_{i} & =a(t) \bar{N}(t)\left(\frac{1}{a} \partial_{i} s+n_{i}^{T}\right),
\end{aligned}
$$

where $\zeta$ is the curvature perturbation and all perturbations carry a temporal and spatial dependence. We have made use of the scalar-vector-tensor decomposition of the metric and scalarvector decomposition of the lapse with

$$
\partial^{i} h_{i}^{T}=0, \quad \partial^{i} h_{i j}^{T T}=0=\partial^{j} h_{i j}^{T T}, \quad \partial^{i} n_{i}^{T}=0 .
$$


We choose to define every derivative in the decomposition of the perturbation with a factor of $a$, such that the combination $a d x$ appears everywhere.

Although apparently linear, the field perturbations contain in principle perturbations to all orders. For instance,

$$
\varphi=\lambda \varphi^{(1)}+\lambda^{2} \varphi^{(2)}+\mathcal{O}\left(\lambda^{3}\right), \quad \lambda \ll 1,
$$

where $\lambda$ indicates the order in perturbations. Similar expansions hold for the other perturbations in (2.5). Note that the background value of $N_{i}$ is zero $\left(s, n_{i}^{T}\right.$ are of $\left.\mathcal{O}(\lambda)\right)$, since we are describing perturbations on top of a FLRW spacetime. Moreover, having $\bar{N}(t)$ as a background for $g_{00}$ allows us to rescale time. For example, conformal time would be defined by setting $\bar{N}(t) \rightarrow a(\eta)$, thus having $\bar{N}(t) d t \rightarrow a(\eta) d \eta$, with $\eta$ being conformal time.

\section{Gauge dependence and gauge invariant perturbations}

As mentioned above the gauge freedom in General Relativity corresponds to the fact that different mappings can be chosen between the physical and background spacetime. Changing the mapping is then referred to as a gauge transformation. If $x^{\mu}$ is the vector field associated with one mapping, then a different gauge choice $x^{\mu}+\xi^{\mu}$ transforms a quantity $Q$ according to $[7,8]$

$$
Q \rightarrow e^{\mathcal{L}_{\xi}} Q
$$

where $\mathcal{L}_{\xi}$ is the Lie derivative along $\xi$. The 4 -vector $\xi^{\mu}$ contains all orders in perturbations, $\xi^{\mu} \equiv \lambda \xi_{(1)}^{\mu}+\frac{1}{2} \lambda^{2} \xi_{(2)}^{\mu}+\mathcal{O}\left(\lambda^{3}\right) . \xi^{\mu}$ can be separated in a temporal and a spatial part, which can be written as

$$
\xi^{\mu}=\left(\xi^{0}, \xi^{i}\right)=\left(\xi^{0}, \frac{1}{a} \partial^{i} \xi+\xi^{i(T)}\right)
$$

where $\xi^{i(T)}$ is the transverse part of the spatial $\xi^{i}\left(\partial_{i} \xi^{i(T)}=0\right)$.

\subsection{Gauge transformations of metric and field fluctuations}

We now study more precisely how the scalar and metric field in (2.1) transform under the gauge transformation (3.1). We consider the gauge transformations up to second order in $\lambda$. For simplicity we only look at gauge transformations of the scalar degrees of freedom $\varphi$ and $\zeta$, which transform under the temporal gauge parameter $\xi^{0}$. We are not interested in second order perturbations of the other scalars $n$ and $\nabla^{2} s$, because they are constraint fields that can be eliminated from the action by solving the constraint equations. Alternatively, they can be decoupled from scalar perturbations in a procedure [6] that constructs the gauge invariant action including gauge invariant constraints. Also, we neglect vector and tensor modes and spatial derivatives which are unimportant on long wavelengths.

For the scalar field $\Phi$ in (2.1) the gauge transformation (3.1) acts on the perturbation as

$$
\varphi \rightarrow \varphi+\dot{\phi} \bar{N} \xi^{0}+\frac{1}{2}\left(\ddot{\phi} \bar{N} \xi^{0}+\dot{\phi}\left(\bar{N} \xi^{0}\right)^{\cdot}+2 \dot{\varphi}\right) \bar{N} \xi^{0}+\mathcal{O}\left(\xi^{i}, \partial_{i} \xi^{0}, \partial_{i} \varphi\right)
$$

Here the dotted derivative denotes a reparametrization invariant time derivative $\dot{\phi}=\frac{d}{N d t} \phi$, such that it is easy to rescale time, for example conformal time by setting $\bar{N}(t) \rightarrow a(\eta)$. Moreover, the spatial derivatives appear with a factor of $a$. At higher order we have only shown quadratic terms containing (temporal derivatives of) $\xi^{0}$. Other terms include $\xi^{i}$ and/or spatial derivatives of $\varphi$ or $\xi^{0}$, which, as we mentioned, have been neglected. 
Similarly, the metric tensor transforms under (3.1). If we consider the curvature perturbation $\zeta$ then

$$
2 \zeta \rightarrow 2 \zeta+2 H \bar{N} \xi^{0}+\bar{N} \xi^{0}\left[2 \dot{\zeta}+\dot{H} \bar{N} \xi^{0}+H\left(\bar{N} \xi^{0}\right)^{\cdot}\right]+\mathcal{O}\left(\xi^{i}, \partial_{i} \xi^{0}, \partial_{i} \zeta\right)
$$

Here $H \equiv \dot{a} / a$. Note that $\zeta$ only transforms under temporal gauge transformations $t \rightarrow t+\xi^{0}$ at linear order in perturbations. Also here we have not explicitly written other higher order terms that include $\xi^{i}$ and/or spatial derivatives of $\zeta$ or $\xi^{0}$. These terms appear when projecting out the gauge transformation of $\zeta$. The precise transformations of $\zeta$ and $\varphi$ can be found, for example, in Refs. [7, 8] or [9] (the latter does not include the spatial gauge transformation $\xi^{i}$ ).

\subsection{Constructing gauge invariant variables}

From Eqs. (3.3) and (3.4) a combination can be formed which is gauge invariant under temporal gauge transformations to first order. This combination is

$$
w \equiv 2 \frac{H}{\dot{\phi}} \varphi-2 \zeta
$$

and is called the comoving curvature perturbation. This gauge invariant combination of scalar metric and field perturbations appears not to be unique, in the sense that it can be rescaled by any function depending on the background fields $(\dot{\phi}, H)$. These background quantities are by construction fixed and do not induce additional gauge transformations. Thus there are in principle infinitely many gauge invariant combinations. However, a rescaling of a parameter by time dependent functions does not change any physical results. From that point of view the first order gauge invariant variable is unique.

Note that we could have also made a gauge invariant combination of scalar metric or field perturbations with the perturbed constraint fields $n$ or $s$ which also transform under temporal gauge transformations. However, here we are only interested in dynamical gauge invariant variables. The constraint fields in the action (2.3) are nondynamical: they can be solved for and their solutions inserted back into the action. Thus, the only gauge invariant dynamical degree of freedom is the comoving curvature perturbation $w$.

Although gauge invariant to first order in perturbations (governed by the small parameter $\lambda$ ), to second order $w$ changes under the gauge transformation

$$
\begin{aligned}
w & \rightarrow w+\left(\frac{\ddot{\phi}}{H \dot{\phi}}-\frac{\dot{H}}{H^{2}}\right)\left[H^{2}\left(\bar{N} \xi^{0}\right)^{2}+2 \zeta H \bar{N} \xi^{0}\right]+\left[\left(\frac{\ddot{\phi}}{H \dot{\phi}}-\frac{\dot{H}}{H^{2}}\right) H w+\dot{w}\right] \bar{N} \xi^{0}+\mathcal{O}\left(\xi^{i}, \partial_{i} \xi^{0}, \partial_{i} w\right) \\
& \equiv w+\Delta_{2, \zeta}^{\xi} w
\end{aligned}
$$

where the second order gauge transformation of $w$ is $\Delta_{2, \zeta}^{\xi} w$, as in Ref. [10], with an additional subscript $\zeta$ which indicates the gauge transformation involves terms $\propto \zeta$.w can be made gauge invariant to second order by adding quadratic perturbations to its definition. For example, we know that to first order $\zeta$ changes under a gauge transformation as $\zeta \rightarrow \zeta+H \bar{N} \xi^{0}$. Therefore we can define

$$
\begin{aligned}
W_{\varphi} & =w-\left[\left(\frac{\ddot{\phi}}{H \dot{\phi}}-\frac{\dot{H}}{H^{2}}\right)\left(\zeta^{2}+w \zeta\right)+\frac{1}{H} \dot{w} \zeta\right] \\
& =w-F_{\zeta}[w, \zeta],
\end{aligned}
$$


which is gauge invariant to second order ${ }^{1}$. This gauge invariant variable is related by a factor $H / \dot{\phi}$ to the gauge invariant field perturbation on uniform curvature hypersurfaces [8, 11], as one can construct it by fixing the vector field $\xi^{\mu}$ at each order such that $\zeta=0$. Here, we have constructed the gauge invariant variable by demanding that the gauge transformation at each order is countered by appropriate terms. We emphasize that we do not want to use any gauge fixing in this work. Rather, we wish to maintain all fields and their perturbations, and eliminate any unphysical degrees of freedom by using gauge invariant variables.

Alternatively we could have countered the temporal gauge dependence in Eq. (3.6) by quadratic terms in $\varphi$ which transform as $\varphi \rightarrow \varphi+\dot{\phi} \bar{N} \xi^{0}$. In that case it is useful to replace $\zeta=\frac{H}{\dot{\phi}} \varphi-\frac{1}{2} w$ in the second order gauge transformation of $w(3.6)$ such that

$$
\begin{aligned}
w & \rightarrow w+\left(\frac{\ddot{\phi}}{H \dot{\phi}}-\frac{\dot{H}}{H^{2}}\right)\left[H^{2}\left(\bar{N} \xi^{0}\right)^{2}+2 \frac{H}{\dot{\phi}} \varphi H \bar{N} \xi^{0}\right]+\dot{w} \bar{N} \xi^{0}+\mathcal{O}\left(\xi^{i}, \partial_{i} \xi^{0}, \partial_{i} w\right) \\
& \equiv w+\Delta_{2, \varphi}^{\xi} w .
\end{aligned}
$$

$\Delta_{2, \varphi}^{\xi} w$ here means the second order gauge transformation of $w$ involving $\varphi$. Of course the gauge transformation here is the same as in Eq. (3.6), i.e. $\Delta_{2, \varphi}^{\xi} w=\Delta_{2, \zeta}^{\xi} w$, because we have merely rewritten the gauge transformation in terms of $\varphi$. Now we can define another variable

$$
\begin{aligned}
W_{\zeta} & =w-\left[\left(\frac{\ddot{\phi}}{H \dot{\phi}}-\frac{\dot{H}}{H^{2}}\right) \frac{H^{2}}{\dot{\phi}^{2}} \varphi^{2}+\frac{1}{\dot{\phi}} \dot{w} \varphi\right] \\
& =w-F_{\varphi}[w, \varphi],
\end{aligned}
$$

which is gauge invariant to second order as well (see footnote 1 ). In the literature $[8,11]$ this variable is constructed by fixing the gauge such that $\varphi=0$ at each order, and is therefore called the curvature perturbation on uniform field hypersurfaces.

In principle we could have picked any combination of quadratic perturbations in $\zeta$ and $\varphi$ to balance the second order gauge dependence of $w$. The advantage of the above variables is that they reduce to the linear perturbations if one of the scalar perturbations is set to zero. For example, in the case where $\zeta=0, W_{\varphi} \rightarrow 2 \frac{H}{\dot{\phi}} \varphi$, or when $\varphi=0$, the other gauge invariant variable $W_{\zeta} \rightarrow-2 \zeta$. As it turns out, this makes it very useful to find the gauge invariant action at third order in terms of these variables. We will discuss this in the next section.

It is obvious that the two gauge invariant variables $W_{\varphi}$ and $W_{\zeta}$ are not equal at second order. Their difference can be expressed in terms of a gauge invariant second order part. For example, substituting the equality $\zeta=\frac{H}{\dot{\phi}} \varphi-\frac{1}{2} w$ in the definition of $W_{\varphi}(3.7)$, we find

$$
\begin{aligned}
W_{\varphi} & =W_{\zeta}+\frac{1}{4 \dot{\phi}}\left(\frac{\dot{\phi}}{H}\right) W_{\zeta}^{2}+\frac{1}{2} \frac{1}{H} W_{\zeta} \dot{W}_{\zeta} \\
& =W_{\zeta}+Q\left(W_{\zeta}, W_{\zeta}\right) .
\end{aligned}
$$

So, the difference between the two gauge invariant variables is quadratic in $W_{\zeta}^{2}$ and its derivatives, and is therefore gauge invariant by itself. For clarification, at second order $W_{\zeta}^{2}=W_{\varphi}^{2}$,

\footnotetext{
${ }^{1}$ Here we have not written terms with vectors, tensors and spatial derivatives of $w$ which are unimportant on superhorizon scales. The complete second order gauge invariant variables can be found in, for example, [8].
} 
so Eq. (3.10) gives a nonlinear relation between the different second order gauge invariant variables. Both gauge invariant variables can be called 'physical' degrees of freedom, in the sense that they do not depend on the unphysical gauge degrees of freedom. The question is if the variables describe the same physics. One could imagine that, since the variables are not equal, the 2-point and 3-point functions are also different and this may give different results. Of course, in order to calculate the 2-point and 3-point functions and describe the dynamics of the gauge invariant variables, one should study the action for them, which is what we do next.

\section{The gauge invariant action for cosmological perturbations}

\subsection{Gauge invariance at zeroth order}

The starting point is the Einstein frame action (2.1), which is manifestly covariant. Now one can insert the ADM metric (2.2) and one finds - up to boundary terms - the action (2.3). Although the general covariance is not manifest in this action, it is still present. We have merely decomposed the metric $g_{\mu \nu}$ in separate parts, but as a whole it still transforms as a tensor.

Now we insert perturbations on top of a fixed homogeneous, isotropic and expanding background. If we consider the background alone, the action is

$$
S^{(0)}=\int d^{3} x d t \bar{N} a^{3}\left\{-3 H^{2}+\frac{1}{2} \dot{\phi}^{2}-V(\phi)\right\} .
$$

This action is trivially covariant, in the sense that the background quantities transform at zeroth order under coordinate transformations, such that the background fields are fixed functions of the coordinates. In other words, if $a=a(t)$ in one coordinate system, than $a=a(\tilde{t})$ in another coordinate system.

The hamiltonian constraint, momentum equation and field equation for the background are found by varying the action with respect to the $\bar{N}, a$ and $\phi$, respectively,

$$
\begin{aligned}
3 H^{2} & =\frac{1}{2} \dot{\phi}^{2}+V(\phi) \\
2 \dot{H} & =-\dot{\phi}^{2} \\
0 & =\ddot{\phi}+3 H \dot{\phi}+V^{\prime}(\phi) .
\end{aligned}
$$

It turns out that it is useful to define a variable $z$ as

$$
z \equiv \frac{\dot{\phi}}{H}
$$

such that the various slow-roll parameters can be written as,

$$
\begin{aligned}
& \epsilon \equiv-\frac{\dot{H}}{H^{2}}=\frac{1}{2} z^{2} \\
& \eta \equiv \frac{\dot{\epsilon}}{\epsilon H}=2 \frac{\dot{z}}{z H} .
\end{aligned}
$$

Here the same definitions have been used as in [2]. These slow-roll parameters are very useful for finding the dominant contributions to $n$ point functions from the action. 


\subsection{Gauge invariance of quadratic action}

The action to linear order in perturbations vanishes due to the classical background equations of motion. The first nontrivial action of perturbations is the second order action in perturbations. Usually one first eliminates the constraint fields $N$ and $N_{i}$ by solving for them and inserting their solutions back into the action. It is only necessary to do this to first order in perturbations since the second order solutions multiply the classical equations of motion. If we consider only the scalar fluctuations $\zeta$ and $\varphi$, the resulting second order action looks schematically like

$$
S^{(2)}(\zeta, \varphi)=\int d^{3} x d t \bar{N} a^{3}\left\{A \mathcal{O}\left(\zeta^{2}\right)+B \mathcal{O}(\zeta \varphi)+C \mathcal{O}\left(\varphi^{2}\right)\right\}
$$

where $\zeta$ and $\varphi$ are now linear in the perturbation parameter $\lambda$, such that the action is of order $\lambda^{2}$. With the indication $\mathcal{O}\left(\zeta^{2}\right)$ we mean all terms of order $\zeta^{2}$, including $\dot{\zeta}^{2}, \zeta \dot{\zeta}, \partial_{i} \zeta \partial^{i} \zeta$ and possible mixings. A similar reasoning applies to the $\mathcal{O}\left(\varphi^{2}\right)$ and $\mathcal{O}(\zeta \varphi)$ terms. The explicit form (4.5) can be found in e.g. [12] or [6].

As was mentioned in Sec. 2 (and shown explicitly in Sec. 3), the perturbations $\zeta$ and $\varphi$ transform under linear gauge transformations, specifically under temporal gauge transformations. Therefore, the variables $\zeta$ and $\varphi$ do not separately have a physical meaning, as they depend on our choice of coordinate system. The combination $w$ in Eq. (3.5) is on the other hand gauge invariant, and if we express the schematic action (4.5) in terms of this $w$, one obtains [12]

$$
S^{(2)}(w)=\int d^{3} x d t \bar{N} a^{3} \frac{1}{4} z^{2}\left\{\frac{1}{2} \dot{w}^{2}-\frac{1}{2}\left(\frac{\partial_{i} w}{a}\right)^{2}\right\},
$$

up to total derivative terms. $z$ was defined in Eq. (4.3). Apart from $w$, the action also contains the transverse, traceless metric perturbation $h_{i j}$, in short the graviton, which is automatically gauge invariant to first order. ${ }^{2}$

The action (4.6) is now manifestly gauge invariant up to second order in perturbations: the variables are diffeomorphism invariant up to a first order in coordinate reparametrizations, and can therefore be considered physical, and the background fields are trivially gauge invariant. One can now do the usual steps of rescaling $w$ to a variable $v=\frac{1}{2} a^{\frac{3}{2}} \frac{\dot{\phi}}{H} w$, the Mukhanov variable, such that the action becomes that of a harmonic oscillator with a time dependent mass. One can then quantize $v$ with the usual expansion in creation and annihilation operators, solve for the mode functions in, for example, an inflationary background, and calculate the power spectrum for those perturbations that remain constant on superhorizon scales (which coincides with the comoving curvature perturbation $w$ on superhorizon scales during slow-roll inflation).

\subsection{Gauge invariance of cubic action}

In order to study non-Gaussianities in the Cosmic Microwave Background radiation (CMB), $n$ point functions such as the primordial bispectrum or trispectrum must be derived. The 3-point functions are found from the third order action in cosmological perturbations. Of course, the

\footnotetext{
${ }^{2}$ Instead of solving for the constraint fields, it is also possible to keep the constraint fields and perturb them to second order [6]. After decoupling them from the dynamical degrees of freedom there are an additional 4 non-dynamical degrees of freedom in the second order action, which are gauge invariant as well. Varying the action with respect to these degrees of freedom gives the first order solutions of the constraint fields. Thus, out of 11 degrees of freedom in the metric and scalar field, we are left with 3 dynamical and 4 constraint degrees of freedom. The 4 gauge degrees of freedom have thus been eliminated from the action.
} 
physical bispectrum is only found when the perturbations in the (tree-level) cubic action are physical, i.e gauge invariant.

Schematically, the third order action in $\zeta$ and $\varphi$ takes the form

$$
S^{(3)}(\zeta, \varphi)=\int d^{3} x d t \bar{N} a^{3}\left\{A \mathcal{O}\left(\zeta^{3}\right)+B \mathcal{O}\left(\zeta^{2} \varphi\right)+C \mathcal{O}\left(\zeta \varphi^{2}\right)+D \mathcal{O}\left(\varphi^{3}\right)\right\}
$$

Again, one can find this action by dropping vector and tensor perturbations and solving the constraint equations to first order in perturbations. The third order solutions multiply the background equations of motion, and the second order solutions multiply the Hamiltonian and momentum constraint evaluated at first order. The $A \mathcal{O}\left(\zeta^{3}\right)$ and $D \mathcal{O}\left(\varphi^{3}\right)$ terms were first derived in Ref. [1], and for completeness we give the terms here explicitly. First the $A \mathcal{O}\left(\zeta^{3}\right)$ terms up to temporal and spatial boundary terms:

$$
\begin{aligned}
S^{(3)}(\zeta)= & \int d^{3} x d t \bar{N} a^{3}\left\{-\left(\zeta^{2}+2 \zeta \frac{\dot{\zeta}}{H}\right) \frac{\nabla^{2} \zeta}{a^{2}}-\left(\zeta+\frac{\dot{\zeta}}{H}\right)\left(\frac{\partial_{i} \zeta}{a}\right)^{2}+\frac{3}{2} z^{2} \zeta \dot{\zeta}^{2}-\frac{1}{2} \frac{z^{2}}{H} \dot{\zeta}^{3}\right. \\
& \left.+\frac{1}{2}\left(3 \zeta-\frac{\dot{\zeta}}{H}\right)\left[\frac{\partial_{i} \partial_{j} \psi}{a^{2}} \frac{\partial_{i} \partial_{j} \psi}{a^{2}}-\left(\frac{\nabla^{2} \psi}{a^{2}}\right)^{2}\right]-2 \frac{\partial_{i} \psi}{a} \frac{\partial_{i} \zeta}{a} \frac{\nabla^{2} \psi}{a^{2}}\right\},
\end{aligned}
$$

where

$$
\frac{\nabla^{2} \psi}{a^{2}}=-\frac{\nabla^{2}}{a^{2}} \frac{\zeta}{H}+\frac{1}{2} z^{2} \dot{\zeta}
$$

Secondly the $D \mathcal{O}\left(\varphi^{3}\right)$ terms:

$$
\begin{aligned}
S^{(3)}(\varphi)= & \int d^{3} x d t \bar{N} a^{3}\left\{-\frac{1}{4} z \dot{\varphi}^{2} \varphi-\frac{1}{4} z\left(\frac{\partial_{i} \varphi}{a}\right)^{2} \varphi-\dot{\varphi} \frac{\partial_{i} \chi}{a} \frac{\partial_{i} \varphi}{a}\right. \\
& +\frac{1}{4} z\left[\varphi\left(\frac{\nabla^{2} \chi}{a^{2}}\right)^{2}-\varphi\left(\frac{\partial_{i} \partial_{j} \chi}{a^{2}}\right)\left(\frac{\partial_{i} \partial_{j} \chi}{a^{2}}\right)\right] \\
& \left.+\left[\frac{1}{8} H^{2} z^{3}\left(3-\frac{1}{2} z^{2}\right)-\frac{1}{4} z V^{\prime \prime}-\frac{1}{6} V^{\prime \prime \prime}\right] \varphi^{3}+\frac{1}{4} z^{3} H \varphi^{2} \dot{\varphi}+\frac{1}{4} z^{2} \varphi^{2} \frac{\nabla^{2} \chi}{a^{2}}\right\}
\end{aligned}
$$

where

$$
\frac{\nabla^{2} \chi}{a^{2}}=\frac{1}{2} z^{2}\left(-\frac{\varphi}{z}\right)
$$

The $A \mathcal{O}\left(\zeta^{3}\right)$ terms have been derived for generalized scalar theories in Ref. [2], see also [3].

\subsubsection{Manifest gauge invariance: cubic action for $W_{\varphi}$}

The action (4.7) does not appear to be covariant due to the gauge dependence of $\zeta$ and $\varphi$. However, we know that the complete action is covariant, and should be covariant to this order as well. To make this more manifest, we can try to express the cubic action in terms of the linear gauge invariant variable $w$, since the third order terms in (4.7) only transform under linear gauge transformations. In doing so we have several possibilities.

The first option is to eliminate all $\varphi$ dependence in the action by replacing

$$
\varphi=\frac{1}{2} z w+z \zeta,
$$


where again $z=\dot{\phi} / H$ such that

$S^{(3)}=\int d^{3} x d t \bar{N} a^{3}\left\{D \mathcal{O}\left(\left(\frac{1}{2} z w\right)^{3}\right)+E \mathcal{O}\left(\zeta^{3}\right)+F \mathcal{O}\left(\zeta^{2} w\right)+G \mathcal{O}\left(\zeta w^{2}\right)\right\}=S_{\mathrm{GI}}^{(3)}(w)+S_{\mathrm{GD}}^{(3)}(\zeta)$.

The first part $S_{\mathrm{GI}}^{(3)}(w)$ now only depends on $\mathcal{O}\left(w^{3}\right)$ terms, and is therefore explicitly gauge invariant at third order in $\lambda$. The second part $S_{\mathrm{GD}}^{(3)}(\zeta)$ is on the other hand gauge dependent, because it contains cubic terms $\zeta^{3}, \zeta^{2} w$ and $\zeta w^{2}$ that cannot balance each other's gauge transformations. However, the original action (2.1) is diffeomorphism invariant, and this gauge dependence should somehow cancel. One should not forget that also the second order action changes under a gauge transformation. Although (4.6) is gauge invariant to first order in $\lambda$, to second order in gauge transformations it is not. The gauge transformation (3.6) thus generates third order terms in $\lambda$ from the second order action (4.6). Following Rigopoulos [10], under a second order gauge transformation of $w$,

$$
S^{(2)}(w) \rightarrow S^{(2)}(w)+\int d^{3} x d t \bar{N} a^{3}\left\{\frac{1}{a^{3}} \frac{\delta S^{(2)}(w)}{\delta w} \Delta_{2, \zeta}^{\xi} w\right\}
$$

where $\Delta_{2, \zeta}^{\xi} w$ was defined in (3.6). In order to keep the general covariance at each order in perturbation theory, this second order gauge transformation of $S^{(2)}(w)$ must be balanced by appropriate linear gauge transformations of $S^{(3)}$. This means that the gauge dependent terms in Eq. (4.13) must be proportional to the first order equations of motion, such that

$$
S_{\mathrm{GD}}^{(3)}(\zeta)=-\int d^{3} x d t \bar{N} a^{3}\left\{\frac{1}{a^{3}} \frac{\delta S^{(2)}(w)}{\delta w} F_{\zeta}[w, \zeta]\right\}
$$

where $F_{\zeta}[w, \zeta]$ is defined in Eq. $(3.7)^{3}$. Of course, the gauge dependent part $S_{\mathrm{GD}}^{(3)}(\zeta)$ is precisely such that it counters the second order gauge transformation of $S^{(2)}(w)$ in (4.14). Now it is straightforward to express the action in terms of the second order gauge invariant variable $W_{\varphi}$,

$$
S^{(2)}+S^{(3)}=\int d^{3} x d t \bar{N} a^{3} \frac{1}{4} z^{2}\left\{\frac{1}{2} \dot{W}_{\varphi}^{2}-\frac{1}{2}\left(\frac{\partial_{i} W_{\varphi}}{a}\right)^{2}\right\}+S_{\mathrm{GI}}^{(3)}\left(W_{\varphi}\right) .
$$

Thus, we have found the third order action which is manifestly gauge invariant up to second order in gauge transformations. The dynamical scalar degree of freedom in this action is $W_{\varphi}$, where $\frac{1}{2} z W_{\varphi}$ is the field perturbation on uniform curvature hypersurfaces. We haven't mentioned explicitly what is $S^{(3)}\left(W_{\varphi}\right)$, that is, what are the gauge invariant cubic vertices for $W_{\varphi}$ needed for the calculations of non-Gaussianities. The easiest way to find this is to set $\zeta=0$ from the start. In that case $W_{\varphi}$ coincides with $\frac{2}{z} \varphi$ and

$$
S_{\mathrm{GI}}^{(3)}\left(W_{\varphi}\right) \stackrel{\zeta \rightarrow 0}{\longrightarrow} \int d^{3} x d t \bar{N} a^{3}\left\{D \mathcal{O}\left(\varphi^{3}\right)\right\}
$$

\footnotetext{
${ }^{3}$ This implies that the terms of $\mathcal{O}\left(\zeta^{3}\right)$ should drop out in the third order action (4.7) after the replacement of $\varphi(4.12)$.
} 
Hence, $S_{\mathrm{GI}}^{(3)}\left(W_{\varphi}\right)$ can be found immediately after the replacement $\varphi \rightarrow \frac{1}{2} z W_{\varphi}$ in the $D \mathcal{O}\left(\varphi^{3}\right)$ terms of (4.7) [10]. Using these terms from [1] the result is

$$
\begin{aligned}
S_{\mathrm{GI}}^{(3)}\left(W_{\varphi}\right)= & \int d^{3} x d t \frac{\bar{N} a^{3}}{8}\left\{\frac{1}{2} z^{4}\left[-\frac{1}{2} \dot{W}_{\varphi}^{2} W_{\varphi}-\frac{1}{2}\left(\frac{\partial_{i} W_{\varphi}}{a}\right)^{2} W_{\varphi}+\dot{W}_{\varphi}\left(\frac{\partial_{i}}{\nabla^{2}} \dot{W}_{\varphi}\right) \partial_{i} W_{\varphi}\right]\right. \\
& +\frac{1}{16} z^{6}\left[\dot{W}_{\varphi}^{2} W_{\varphi}-\left(\frac{\partial_{i} \partial_{j}}{\nabla^{2}} \dot{W}_{\varphi}\right)\left(\frac{\partial_{i} \partial_{j}}{\nabla^{2}} \dot{W}_{\varphi}\right) W_{\varphi}\right] \\
& +\left[\frac{1}{8} H z^{6}-\frac{1}{2} z^{3} \dot{z}\right] W_{\varphi}^{2} \dot{W}_{\varphi}+\frac{1}{2} z^{3} \dot{z} W_{\varphi}\left(\frac{\partial_{i}}{\nabla^{2}} \dot{W}_{\varphi}\right) \partial_{i} W_{\varphi} \\
& \left.+\left[-\frac{1}{4} z^{2} \dot{z}^{2}+\frac{1}{4} H z^{5} \dot{z}+\frac{1}{8} H^{2} z^{6}\left(3-\frac{1}{2} z^{2}\right)-\frac{1}{4} z^{4} V^{\prime \prime}-\frac{1}{6} z^{3} V^{\prime \prime \prime}\right] W_{\varphi}^{3}\right\} .
\end{aligned}
$$

This demonstrates the usefulness of the field perturbation on uniform curvature hypersurfaces $\frac{1}{2} z W_{\varphi}$ in combination with the uniform curvature gauge $\zeta=0$. The cubic action (4.18) can be simplified further by doing partial integrations of the third and last lines and using the background equations of motion (4.2),

$$
\begin{aligned}
S_{\mathrm{GI}}^{(3)}\left(W_{\varphi}\right)= & \int d^{3} x d t \frac{\bar{N} a^{3}}{8}\left\{\frac{1}{2} z^{4}\left[-\frac{1}{2} \dot{W}_{\varphi}^{2} W_{\varphi}-\frac{1}{2}\left(\frac{\partial_{i} W_{\varphi}}{a}\right)^{2} W_{\varphi}+\dot{W}_{\varphi}\left(\frac{\partial_{i}}{\nabla^{2}} \dot{W}_{\varphi}\right) \partial_{i} W_{\varphi}\right]\right. \\
& \left.+\frac{1}{16} z^{6}\left[\dot{W}_{\varphi}^{2} W_{\varphi}-\left(\frac{\partial_{i} \partial_{j}}{\nabla^{2}} \dot{W}_{\varphi}\right)\left(\frac{\partial_{i} \partial_{j}}{\nabla^{2}} \dot{W}_{\varphi}\right) W_{\varphi}\right]-\frac{1}{2} z^{2} \dot{W}_{\varphi} W_{\varphi}^{2}\left[\frac{\dot{z}}{z H}\right]\right\} .
\end{aligned}
$$

In Eq. (4.19) different orders in slow-roll can easily be distinguished. Using the slow-roll parameters (4.4) it is clear that the first line contains terms of order $\epsilon^{2}$, whereas the second line is subleading in slow-roll.

In the derivation of the gauge invariant action for $W_{\varphi}$ we have set $\zeta=0$. What happens if we would have taken $\varphi=0$ from the start? In that case the third order action (4.7) contains only terms $A \mathcal{O}\left(\zeta^{3}\right)$, and $W_{\varphi}$ is a nonlinear expression in $\zeta$. Therefore the third order action must contain terms proportional to the first order equation of motion for $\zeta$, which can be absorbed in the second order action by identifying $W_{\varphi}(\varphi=0)$. These terms proportional to the equation of motion were identified in [1]. It was shown that after the field redefinition $\zeta \rightarrow \frac{1}{2} W_{\varphi}(\varphi=0)$, the cubic action for $\varphi$ is obtained by replacing $W_{\varphi} \rightarrow \frac{2 H}{\dot{\phi}} \varphi$. In our language of gauge invariance, we redefine $\zeta$ to the gauge invariant variable $W_{\varphi}$ (in the gauge $\varphi=0$ ), then restore the dependence on $\varphi$ in $W_{\varphi}$, and finally set $\zeta=0$ to get the action for $\mathcal{O}\left(\varphi^{3}\right)$ terms.

\subsubsection{Manifest gauge invariance: cubic action for $W_{\zeta}$}

In the previous section we found the manifestly gauge invariant cubic action in terms of $W_{\varphi}$, related to the field perturbation on uniform curvature hypersurfaces. Just as well we could have constructed the gauge invariant action for $W_{\zeta}$, the curvature perturbation on uniform field hypersurfaces. Starting point is again the schematic third order action (4.7) and we follow the same steps as in the previous part. Instead of eliminating $\varphi$ in terms of $w$ and $\zeta$ as in Eq. (4.12), we eliminate $\zeta$

$$
\zeta=\frac{1}{z} \varphi-\frac{1}{2} w .
$$

As before, the third order action $S^{(3)}$ can then be separated in a gauge invariant part depending only on $\mathcal{O}\left(w^{3}\right)$ terms, and a gauge non-invariant part depending on $\varphi$. Of course, this gauge 
dependent part must again balance the gauge transformation of $S^{(2)}(4.14)$. Therefore

$$
\begin{aligned}
S^{(3)} & =\int d^{3} x d t \bar{N} a^{3}\left\{A \mathcal{O}\left(\left(-\frac{1}{2} w\right)^{3}\right)+E^{\prime} \mathcal{O}\left(\varphi^{3}\right)+F^{\prime} \mathcal{O}\left(\varphi^{2} w\right)+G^{\prime} \mathcal{O}\left(\varphi w^{2}\right)\right\} \\
& =\tilde{S}_{\mathrm{GI}}^{(3)}(w)+\tilde{S}_{\mathrm{GD}}^{(3)}(\varphi) \\
& =\tilde{S}_{\mathrm{GI}}^{(3)}(w)-\int d^{3} x d t \bar{N} a^{3}\left\{\frac{1}{a^{3}} \frac{\delta S^{(2)}(w)}{\delta w} F_{\varphi}[w, \varphi]\right\} .
\end{aligned}
$$

Again we can do a redefinition of $w$ to the second order gauge invariant $W_{\zeta}$ using (3.9) such that

$$
S^{(2)}+S^{(3)}=\int d^{3} x d t \bar{N} a^{3} \frac{1}{4} z^{2}\left\{\frac{1}{2} \dot{W}_{\zeta}^{2}-\frac{1}{2}\left(\frac{\partial_{i} W_{\zeta}}{a}\right)^{2}\right\}+\tilde{S}_{\mathrm{GI}}^{(3)}\left(W_{\zeta}\right) .
$$

Thus, following this procedure one obtains the manifestly gauge invariant action at third order expressed in terms of the gauge invariant variable $W_{\zeta}$. The simplest way to find the gauge invariant vertices in $\tilde{S}_{\mathrm{GI}}^{(3)}\left(W_{\zeta}\right)$ is now to set the field perturbation $\varphi=0$. Then, the gauge invariant variable becomes $W_{\zeta}(\varphi=0)=-2 \zeta$, and

$$
\tilde{S}_{\mathrm{GI}}^{(3)}\left(W_{\zeta}\right) \stackrel{\varphi \rightarrow 0}{\longrightarrow} \int d^{3} x d t \bar{N} a^{3}\left\{A \mathcal{O}\left(\zeta^{3}\right)\right\} .
$$

So, if we set $\varphi=0$, the third order action for $\zeta$ immediately gives the gauge invariant action in terms of the curvature perturbation on uniform field hypersurfaces after the replacement $\zeta \rightarrow-\frac{1}{2} W_{\zeta}$. Using the $A \mathcal{O}\left(\zeta^{3}\right)$ terms derived in $[1,2]$,

$$
\begin{aligned}
\tilde{S}_{\mathrm{GI}}^{(3)}\left(W_{\zeta}\right)= & \int d^{3} x d t \frac{\bar{N} a^{3}}{8}\left\{\frac{1}{2} z^{2} W_{\zeta}\left(\frac{\partial_{i} W_{\zeta}}{a}\right)^{2}-\frac{3}{2} z^{2} W_{\zeta} \dot{W}_{\zeta}^{2}+\frac{1}{2} \frac{z^{2}}{H} \dot{W}_{\zeta}^{3}\right. \\
& \left.-\frac{1}{2}\left(3 W_{\zeta}-\frac{\dot{W}_{\zeta}}{H}\right)\left[\frac{\partial_{i} \partial_{j} \psi}{a^{2}} \frac{\partial_{i} \partial_{j} \psi}{a^{2}}-\left(\frac{\nabla^{2} \psi}{a^{2}}\right)^{2}\right]+2 \frac{\partial_{i} \psi}{a} \frac{\partial_{i} W_{\zeta}}{a} \frac{\nabla^{2} \psi}{a^{2}}\right\},
\end{aligned}
$$

where

$$
\frac{\nabla^{2} \psi}{a^{2}}=-\frac{\nabla^{2}}{a^{2}} \frac{W_{\zeta}}{H}+\frac{1}{2} z^{2} \dot{W}_{\zeta}
$$

This demonstrates the convenience of working with the gauge invariant variable $W_{\zeta}$ in combination with the gauge $\varphi=0{ }^{4}$.

\section{Uniqueness of gauge invariant action}

In the previous section two third order actions for cosmological perturbations were derived which were manifestly gauge invariant up to second order in gauge transformations. The general trick is that the gauge dependent parts of the third order action could be absorbed in the second order action, which defined a gauge invariant variable. In Eq. (4.16) the gauge invariant cubic action was expressed in terms of $W_{\varphi}$, in Eq. (4.22) in terms of $W_{\zeta}$. Comparing the gauge invariant

\footnotetext{
${ }^{4}$ In the derivation of (4.24) we have performed several partial integrations with respect to spatial derivatives. The corresponding boundary terms do not contribute to the bispectrum, contrary to the boundary terms for temporal partial integrations, which will be discussed next.
} 
actions (4.16) and (4.22), we see that the parts quadratic in the gauge invariant variables are the same. Thus the tree-level propagator for $W_{\varphi}$ is the same for $W_{\zeta}$. The gauge invariant parts of the action which are cubic in the gauge invariant variables, Eqs. (4.19) and (4.24), appear not to be the same,

$$
S_{\mathrm{GI}}^{(3)}\left(W_{\varphi}\right) \neq \tilde{S}_{\mathrm{GI}}^{(3)}\left(W_{\zeta}\right) .
$$

This implies that the gauge invariant vertices for $W_{\varphi}$ differ from those for $W_{\zeta}$. On the other hand, the gauge invariant cubic vertices originate from the same action. This presents an opportunity to find out exactly how the gauge invariant actions differ.

\subsection{Non-linear transformations}

To illustrate what is the difference between gauge invariant actions for non-linearly related variables, let us take a general action for a second order gauge invariant variable $W_{X}$,

$$
S\left(W_{X}\right)=\int d^{3} x d t \bar{N} a^{3} \frac{1}{4} z^{2}\left\{\frac{1}{2} \dot{W}_{X}^{2}-\frac{1}{2}\left(\frac{\partial_{i} W_{X}}{a}\right)^{2}\right\}+S_{\mathrm{GI}}^{(3)}\left(W_{X}\right) .
$$

This variable is non-linearly related to another second order gauge invariant variable $W_{Y}$,

$$
W_{X}=W_{Y}+Q\left(W_{Y}, W_{Y}\right)
$$

where the $Q\left(W_{Y}, W_{Y}\right)$ is a completely general function quadratic in $W_{Y}$, which can include temporal and/or spatial derivatives of $W_{Y}$. The action for $W_{Y}$ then becomes

$$
\begin{aligned}
S\left(W_{Y}\right) & =\int d^{3} x d t \bar{N} a^{3} \frac{1}{4} z^{2}\left\{\frac{1}{2} \dot{W}_{Y}^{2}-\frac{1}{2}\left(\frac{\partial_{i} W_{Y}}{a}\right)^{2}\right\}+S_{\mathrm{GI}}^{(3)}\left(W_{Y}\right) \\
& +\int d^{3} x d t \bar{N} a^{3}\left\{\frac{1}{a^{3}}\left[\frac{1}{4} a^{3} z^{2} Q \dot{W}_{Y}\right]-\frac{1}{a} \partial_{i}\left[Q \frac{1}{a} \partial_{i} W_{Y}\right]\right\}+\int d^{3} x d t \bar{N} a^{3}\left\{Q \frac{1}{a^{3}} \frac{\delta S^{(2)}\left(W_{Y}\right)}{\delta W_{Y}}\right\},
\end{aligned}
$$

where $\delta S^{(2)} / \delta W_{Y}$ represents the equation of motion following from the quadratic action, i.e.

$$
\frac{1}{a^{3}} \frac{\delta S^{(2)}\left(W_{Y}\right)}{\delta W_{Y}}=\left[-\frac{1}{4} a^{3} z^{2} \dot{W}_{Y}\right]+\frac{1}{a^{2}} \nabla^{2} W_{Y}
$$

These terms are related to the variation of the complete action as

$$
\frac{1}{a^{3}} \frac{\delta S\left(W_{Y}\right)}{\delta W_{Y}}=\frac{1}{a^{3}} \frac{\delta S^{(2)}\left(W_{Y}\right)}{\delta W_{Y}}+\mathcal{O}\left(W_{Y}^{2}\right)=0,
$$

which vanishes by the variational principle. In the third order action the terms $\propto Q \delta S^{(2)} / \delta W_{Y}$ therefore are zero at the cubic level upon inserting the solutions of the equations of motion (5.6), and consequently these terms do not contribute to the 3-point function at tree-level. However, contributions enter in the tree-level action at higher order that can contribute to the 2- and 3 -point function through quantum loops, which we do not consider here.

Inspecting the tree-level cubic gauge invariant action for $W_{Y}(5.4)$ we see that the bulk action $S_{\mathrm{GI}}^{(3)}\left(W_{Y}\right)$ coincides with the gauge invariant cubic action for $W_{X}(5.2)$. In this sense 
the bulk gauge invariant cubic action can be called unique. Thus, the evolution of the 3point function is independent of the choice variables $W_{X}$ or any nonlinearly related variable, characterized by $W_{Y}$.

Of course there are also boundary terms in the action for $W_{Y}$ (5.4). In the in-in or Schwinger-Keldysh formalism they can contribute to the 3-point function. In this formalism an expectation value may be defined as

$$
\begin{aligned}
\left\langle\Omega, t_{\text {in }}|\mathcal{O}(W(t))| \Omega, t_{\text {in }}\right\rangle=\int & {\left[\mathcal{D} W_{+} \mathcal{D} W_{-}\right] \mathcal{O}(W(t)) \rho\left[W_{+}\left(t_{\text {in }}\right), W_{-}\left(t_{\text {in }}\right)\right] } \\
& \times \exp \left(i \int_{t_{\text {in }}}^{t} d t^{\prime}\left(L\left[W_{+}\left(t^{\prime}\right)\right]-L\left[W_{-}\left(t^{\prime}\right)\right]\right)\right) \delta\left[W_{+}(t)-W_{-}(t)\right],
\end{aligned}
$$

where $\rho\left[W_{+}\left(t_{\text {in }}\right), W_{-}\left(t_{\text {in }}\right)\right]$ is the density matrix at initial time $t_{\text {in }}$, which for a pure initial state equals $\Psi^{*}\left[W_{+}\left(t_{\text {in }}\right)\right] \Psi\left[W_{-}\left(t_{\text {in }}\right)\right] . \quad W_{ \pm}$is here a second order gauge invariant variable on the + or - part of the complex in-in contour, which could be, for instance, $W_{X}$ or $W_{Y}$. In general, the operator $\mathcal{O}(W(t))$ depends on both $W_{+}$and $W_{-}$fields. In the simple case of an equal-time 3 -point function, operator ordering is not important, one can drop the \pm subscripts from $W$ 's and $\mathcal{O}(W(t))=W\left(\vec{x}_{1}, t\right) W\left(\vec{x}_{2}, t\right) W\left(\vec{x}_{3}, t\right)$. Coming back to the boundary terms, the spatial ones do not contribute to the 3 -point function. On the other hand, the temporal boundary terms give in general a nonzero contribution.

For cosmological correlation functions (5.7) the initial time is often taken at $t_{0}=-\infty$. Strictly speaking, this is not the correct procedure. As $t \rightarrow-\infty$ the quantum field theory of gravity becomes strongly coupled (that is, in that limit the physical momenta $k / a \rightarrow \infty$ ) and perturbation theory fails. In practice one can define an in-in expectation value by starting from some finite initial time $t_{0}$ at which perturbation theory is well defined. Thus boundary terms at $t_{0}$ can contribute. One can define the initial state for, for instance, the gauge invariant variable $W_{X}$ to be Gaussian. As a consequence the initial state for any other non-linearly related variable $W_{Y}$ is explicitly non-Gaussian. This initial non-Gaussianity is then evolved through the bulk action $S_{\mathrm{GI}}^{(3)}\left(W_{Y}\right)$. It is important to distinguish how much non-Gaussianity is dynamically generated from some Gaussian initial state, and how much comes from a potentially non-Gaussian initial state. For example, if one observes non-Gaussianity for the variable $W_{Y}$, but one defines the initial state for $W_{X}$ to be Gaussian, then some of the final non-Gaussianity finds its origin in the initial non-Gaussianity in $W_{Y}$.

Let us now discuss the contributions to the 3-point function coming from the temporal boundary terms at time $t$. There can be various types of boundary terms. Boundary terms of the type $W^{3}(t)$ cannot contribute to the 3-point function, because the $\delta$-function in Eq. (5.7) forces the "+" and "-" vertices to be equal at time $t$. In fact, these boundary terms do not naturally appear after the transformation to a different gauge invariant variable, as can be seen in (5.4). Nonetheless these terms can appear after additional partial integrations of terms $\propto W^{3}(t)$ and $\propto W^{2}(t) \dot{W}(t)$ in the bulk action. This was exactly done for the action of $W_{\varphi}$ in going from Eq. (4.18) to (4.19), which justifies the use of (4.19) as the gauge invariant cubic action for $W_{\varphi}$.

One has to be careful with other boundary terms such as $\alpha W^{2} \dot{W}$ or $\beta W \dot{W}^{2}$, because they give in general non-negligible contributions to the bispectrum. The reason is that these terms contain the canonical momentum $\Pi_{W}$, which has a nonvanishing commutation relation with $W$. In fact these type of boundary terms generate disconnected parts of the bispectrum. In the 
general example above, it can be seen that the temporal boundary terms in the action for $W_{Y}$ are precisely of the form $S_{\partial}\left(W_{Y}\right)=\int d^{3} x \Pi_{W_{Y}}(t) Q(t)$. Consider now an example where

$$
Q\left(W_{Y}, W_{Y}\right)=\alpha(t) W_{Y}^{2}+\beta(t) W_{Y} \dot{W}_{Y} .
$$

Then using the expectation value as defined in Eq. (5.7) one can compute to lowest (tree-level) order that ${ }^{5}$

$$
\begin{aligned}
\left\langle W_{X}\left(x_{1}\right) W_{X}\left(x_{2}\right) W_{X}\left(x_{3}\right)\right\rangle & =\left\langle W_{Y}\left(x_{1}\right) W_{Y}\left(x_{2}\right) W_{Y}\left(x_{3}\right)\right\rangle \\
& +2 \alpha(t)\left(\left\langle W_{Y}\left(x_{1}\right) W_{Y}\left(x_{2}\right)\right\rangle\left\langle W_{Y}\left(x_{1}\right) W_{Y}\left(x_{3}\right)\right\rangle+\text { sym }\right) \\
& +\beta(t)\left(\left\langle\dot{W}_{Y}\left(x_{1}\right) W_{Y}\left(x_{2}\right)\right\rangle\left\langle W_{Y}\left(x_{1}\right) W_{Y}\left(x_{3}\right)\right\rangle\right. \\
& \left.+\left\langle W_{Y}\left(x_{1}\right) W_{Y}\left(x_{2}\right)\right\rangle\left\langle\dot{W}_{Y}\left(x_{1}\right) W_{Y}\left(x_{3}\right)\right\rangle+\operatorname{sym}\right)
\end{aligned}
$$

where sym stands for other cyclic contributions. In words: the 3-point function for the variable $W_{X}$ is computed using the gauge invariant cubic vertices in $S_{\mathrm{GI}}^{(3)}\left(W_{X}\right)$, the result expressed on the left-hand side, but it is also directly related to the 3-point function for $W_{Y}$, computed using gauge invariant vertices for $W_{Y}$, plus additional disconnected parts coming from the boundary terms, which add up to the right-hand side of (5.10). Note that the relation between the 3-point functions in (5.10) can be immediately derived by inserting the non-linear relation between $W_{X}$ and $W_{Y}$ (5.3) into the left-hand side of (5.10) and using Wick's theorem. Thus in order to compute the 3-point function of one gauge invariant variable, one can use the gauge invariant action for another variable (which may have a more convenient form) and add disconnected pieces according to the non-linear relation.

Since time $t$ in (5.10) is arbitrary, relation (5.10) holds also for $t=t_{\text {in }}$, telling us how are initial non-Gaussianities in the variables $W_{X}$ and $W_{Y}$ related. These variables also define some spacelike hypersurfaces $\Sigma_{W_{X}}$ and $\Sigma_{W_{Y}}$. For example, if the initial state on $\Sigma_{W_{Y}}$ is Gaussian, then the initial non-Gaussianity on $\Sigma_{W_{Y}}$ will be given by the terms multiplying $\alpha$ and $\beta$ in (5.10) evaluated at $t=t_{\mathrm{in}}$.

\subsection{Practical example: different gauge invariant variables}

The general discussion in this section demonstrates that the cubic gauge invariant actions (5.2)(5.4) for different ${ }^{6}$ gauge invariant variables are related, in the sense that they both have the same, unique, bulk action, but they differ by boundary terms and terms proportional to the equation of motion. This is not always obvious. For example, the terms proportional to the equation of motion can be separated, partially integrated, and the remaining terms can be written such that it is not clear that they are total derivative terms. Therefore gauge invariant

\footnotetext{
${ }^{5}$ An alternative way to compute the 3-point correlator (5.10) is by making use of the so-called interaction picture $[1,13]$, where an expectation value to lowest order in perturbation theory is given by

$$
\langle\mathcal{O}(W(t))\rangle=-i \int_{t_{0}}^{t} d t^{\prime}\left\langle\left[\mathcal{O}(W(t)), H_{\mathrm{int}}\left(t^{\prime}\right)\right]\right\rangle .
$$

If for the interaction Hamiltonian $H_{\text {int }}$ one considers the part with the boundary terms in (5.4) only, which are of the form $\Pi_{W}(t) Q(t)$, then it is straightforward to find the disconnected pieces in (5.10) using the canonical commutation relations.

${ }^{6}$ Different here means non-linearly related. This in turn means that the gauge invariant variables coincide at the linear level, but differ at quadratic order.
} 
actions for different, non-linearly related variables can appear very different. To illustrate this, let us now consider a practical example. The gauge invariant variables $W_{\varphi}$ and $W_{\zeta}$ are nonlinearly related as in (3.10), which is an example of (5.3). According to the above discussion, their 3-point functions should therefore be related

$$
\begin{aligned}
\left\langle W_{\varphi}\left(x_{1}\right) W_{\varphi}\left(x_{2}\right) W_{\varphi}\left(x_{3}\right)\right\rangle & =\left\langle W_{\zeta}\left(x_{1}\right) W_{\zeta}\left(x_{2}\right) W_{\zeta}\left(x_{3}\right)\right\rangle \\
& +\frac{1}{2} \frac{\dot{z}}{z H}\left(\left\langle W_{\zeta}\left(x_{1}\right) W_{\zeta}\left(x_{2}\right)\right\rangle\left\langle W_{\zeta}\left(x_{1}\right) W_{\zeta}\left(x_{3}\right)\right\rangle+\operatorname{sym}\right)+\ldots,
\end{aligned}
$$

where the terms of higher order in slow-roll have been neglected. Note that this relation can also be inverted to give the 3-point function of $W_{\zeta}$ in terms $W_{\varphi}$. This is purely based on the non-linear relation between the gauge invariant variables, but it should follow from the actions as well. The gauge invariant actions for $W_{\varphi}$ and $W_{\zeta}$ coincide at the quadratic level, but the cubic actions Eq. (4.19) and (4.24) look very different at first sight. For instance, the action for $W_{\varphi}$ (4.19) is of second order in slow-roll $\left(\epsilon^{2}=\frac{1}{4} z^{4}\right)$, whereas the action for $W_{\zeta}$ (4.24) seems to be of zeroth order. Maldacena [1] showed that it is possible to relate the cubic action for $\varphi$ in the $\zeta=0$ gauge with the cubic action for $\zeta$ in the $\varphi=0$ gauge. The two actions differ by terms proportional to the linear equation of motion (which do not contribute to the tree-level action) and by some boundary terms. When translated to our language of gauge invariant variables, the cubic action for $W_{\varphi}$ (defined on the $\zeta=0$ hypersurface) can be related to $W_{\zeta}$ (defined on the $\varphi=0$ hypersurface), up to boundary terms. After many partial integrations of (4.24), the result is:

$$
\begin{aligned}
\tilde{S}_{\mathrm{GI}}^{(3)}\left(W_{\zeta}\right)= & \int d^{3} x d t \frac{\bar{N} a^{3}}{8}\left\{\frac{1}{2} z^{4}\left[-\frac{1}{2} \dot{W}_{\zeta}^{2} W_{\zeta}-\frac{1}{2}\left(\frac{\partial_{i} W_{\zeta}}{a}\right)^{2} W_{\zeta}+\dot{W}_{\zeta}\left(\frac{\partial_{i}}{\nabla^{2}} \dot{W}_{\zeta}\right) \partial_{i} W_{\zeta}\right]\right. \\
& +\frac{1}{16} z^{6}\left[\dot{W}_{\zeta}^{2} W_{\zeta}-\left(\frac{\partial_{i} \partial_{j}}{\nabla^{2}} \dot{W}_{\zeta}\right)\left(\frac{\partial_{i} \partial_{j}}{\nabla^{2}} \dot{W}_{\zeta}\right) W_{\zeta}\right]-\frac{1}{2} z^{2} \dot{W}_{\zeta} W_{\zeta}^{2}\left[\frac{\dot{z}}{z H}\right] \\
& \left.+Q\left(W_{\zeta}, W_{\zeta}\right) \frac{1}{a^{3}} \frac{\delta S^{(2)}\left(W_{\zeta}\right)}{\delta W_{\zeta}}\right\} \\
= & S_{\mathrm{GI}}^{(3)}\left(W_{\zeta}\right)+\int d^{3} x d t \frac{\bar{N} a^{3}}{8}\left\{Q\left(W_{\zeta}, W_{\zeta}\right) \frac{1}{a^{3}} \frac{\delta S^{(2)}\left(W_{\zeta}\right)}{\delta W_{\zeta}}\right\} .
\end{aligned}
$$

This is precisely the form of the action predicted after insertion of the non-relation relation $W_{\varphi}=W_{\zeta}+Q\left(W_{\zeta}, W_{\zeta}\right)$, as in (5.4), up to boundary terms. The gauge invariant vertices in the bulk action $S_{\mathrm{GI}}^{(3)}$ for $W_{\zeta}$ coincide with those for $W_{\varphi}$. Thus, the cubic gauge invariant action for $W_{\varphi}$ can be called the unique action which separates the different levels of slow-roll. A gauge invariant action in terms of any other gauge invariant variable, e.g. $W_{\zeta}$ can be brought to this unique form after many partial integrations and extracting terms proportional to the equation of motion. The bispectrum for $W_{\zeta}$ can now be computed by making use of the partially integrated bulk action, including possible contributions coming from boundary terms which can give rise to disconnected contributions to 3-point functions, see Eq. (5.10)-(5.11). Alternatively, one can redefine the field $W_{\zeta}$ to the non-linearly related $W_{\varphi}$ and use the action for that gauge invariant variable. The 3-point function for $W_{\zeta}$ is then computed from Eq. (5.11). The non-linear relation between different gauge invariant variables prescribes what this field redefinition should be.

\subsection{Boundary terms, hypersurfaces and observations}

So far we have not shown the boundary terms in (5.12). They were explicitly computed for $\zeta$ (or $W_{\zeta}$ in $\varphi=0$ gauge) in Refs. [14] and [15] for scalar field Lagrangians which are a general function 
$\Phi$ and its kinetic term. Both reach same conclusions: boundary terms with time derivatives of $W_{\zeta}$ contribute to the bispectrum, and the dominant terms in the slow-roll approximation give exactly the same contribution as in Eq. (5.11), which is what one finds after a "field redefinition" to a non-linear variable in $W_{\zeta} \cdot{ }^{7}$ In our language this is nothing more than switching between different gauge invariant variables.

One remark here is that the boundary terms in Refs. [14] and [15] do not disappear completely after redefining $W_{\zeta}$ to a new non-linear variable. On the other hand, Eq. (5.4) suggests that all boundary terms are incorporated after switching to a non-linear variable. This must be so because under a non-linear transformation 3-point functions of different variables are related as (5.10), irrespective of a specific action. When describing now different gauge invariant variables with their corresponding actions, the same relation of the bispectra should follow from the action, for both the bulk and the boundary. The origin of these additional boundary terms not removed by the field redefinition may reside in additional boundary terms of the quadratic action. The form of the quadratic action in (4.6) is only reached after several partial integrations, which generate additional second order boundary terms. Moreover, the original, unperturbed ADM action (2.3) also contains several spatial and temporal boundary terms that in principle contribute at every order. Together these boundary terms must add up to a gauge invariant second order boundary term, expressed in the linear $w$, since after all the original starting point is the covariant action (2.1). Now, under a non-linear field transformation these boundary terms will generate also cubic boundary terms, which may balance the extra boundary terms mentioned before.

A different way to see this is to come back to the procedure of finding the gauge invariant cubic action, outlined in Sec. 4.3. Here the non-linear gauge transform of the second order bulk action was balanced by gauge dependent terms in the third order action, which are proportional to the linear equation of motion. This in turn defined a second order gauge invariant variable ( $W_{\varphi}$ or $W_{\zeta}$ depending on the procedure). Similarly, also the second order boundary terms transform under non-linear gauge transformations. They can be written in a gauge invariant way after incorporating the gauge dependent third order boundary terms.

In spite of these remarks the conclusion of [14] and [15] still stands: the dominant contribution to the bispectrum coming from the boundary terms for $W_{\zeta}$ is taken into account by switching to a different gauge invariant variable $W_{\varphi}$ and using (5.11). This can be very useful. Ultimately we are interested in describing correlation functions of $W_{\zeta}$ (the second order gauge invariant comoving curvature perturbation), because this is the field that is conserved on superhorizon scales and forms the initial fluctuations in the gravitational potential that are believed to form the seeds of structure formation and the observed temperature fluctuations in the CMB. The correct gauge invariant cubic action to use is therefore Eq. (4.24). However, this action does not clearly separate the dominant contributions in the slow-roll approximation. Before horizon crossing it is therefore much more useful to work with the non-linearly related variable $W_{\varphi}$, for which it is straightforward to determine the dominant vertices. Note that one has to be careful concerning the initial state: if the initial state for $W_{\zeta}$ is Gaussian at $t_{0}$, then for $W_{\varphi}$ it will be automatically non-Gaussian. After horizon crossing the full action for $W_{\zeta}$ should be

\footnotetext{
${ }^{7}$ In fact, in Ref. [15] the procedure is slightly different than stated above. After partial integrations Burrage et al. do not keep any boundary terms proportional $W_{\zeta}^{2} \dot{W}_{\zeta}$. The reason is precisely that these would generate disconnected parts of the 3-point function. The partial integrations performed are only the "allowed" ones: those that do not contribute to the bispectrum at all, or those that are slow-roll suppressed contributions. The field redefinition is slightly different in their work, and it does not coincide with the variable $W_{\varphi}$ (note: all computations are performed in the comoving, $\varphi=0$, gauge.)
} 
used, as this variable is conserved on superhorizon scales.

Finally some remarks about non-Gaussianity and observations. As argued, the variable to use to calculate the primordial power spectrum and non-Gaussianity is $W_{\zeta}$. The CMB power spectrum, and possible non-Gaussianities there, are namely created by fluctuations in the gravitational potential. The universe reheats at a $\varphi=0$ (more generally $\delta \rho=0$ ) hypersurface where photons decouple. These photons carry the information of the gravitational fluctuations towards us. However, we observe these photons via satellites such as WMAP or Planck, that are to a good approximation freely-falling observers for which $\zeta=0$, i.e. observations are made on zero curvature hypersurfaces. Choosing a certain gauge invariant variable to work with, is in essence nothing else but choosing the hypersurface. ${ }^{8}$ We have seen that different gauge invariant variables are non-linearly related, and therefore their $n$-point functions are related via disconnected pieces. As a consequence, on one hypersurface perturbations may appear nonGaussian, whereas they are in fact Gaussian on a different hypersurface, or vice versa. It is important to distinguish the amount of non-Gaussianity that is generated by the cosmological evolution, and non-Gaussianity that originates from the choice of hypersurface, either initially or at time of observation.

\section{Frame independent cosmological perturbations}

In the first part of this work we have discussed uniqueness of the gauge invariant action for cosmological perturbations with respect to the choice of gauge invariant variables. In this part we consider a different type of uniqueness for the action for cosmological perturbations, namely uniqueness with respect to different conformal frames. Examples are the Einstein frame, Jordan frame or string frame, which are related via field dependent conformal transformations of the metric and redefinitions of the scalar field. Our goal here is to show that at the level of perturbations the (cubic) action can be written in a unique form, which is independent of the Einstein or Jordan frame.

\subsection{Einstein frame and Jordan frame}

So far we have discussed perturbations of the Einstein frame action (2.1). It is also possible to study perturbations in the Jordan frame, where a non-minimal coupling between the scalar field and the Ricci scalar is present. The Jordan frame action is

$$
S_{J}=\int d^{4} x \sqrt{-g_{J}}\left\{\frac{1}{2} R_{J} F\left(\Phi_{J}\right)-\frac{1}{2} g_{J}^{\mu \nu} \partial_{\mu} \Phi_{J} \partial_{\nu} \Phi_{J}-V_{J}\left(\Phi_{J}\right)\right\} .
$$

All subscripts $J$ indicate that the quantities are expressed in the Jordan frame. The function $F\left(\Phi_{J}\right)$ presents the general coupling between the Ricci scalar and the scalar field $\Phi_{J}$. Setting $F\left(\Phi_{J}\right)=1\left(=m_{p l}^{2} \equiv 1 /(8 \pi G)\right)$ takes us back to the minimally coupled case. An example of a model with nonminimal coupling is Higgs inflation $[16,17]$ where $F\left(\Phi_{J}\right)=1+\xi \Phi_{J}^{2}, \xi \gg 1$, and $\Phi_{J}$ is the Higgs field.

\footnotetext{
${ }^{8}$ Albeit the choice of a hypersurface seemingly relies on a gauge variant concept of setting e.g. $\varphi=0$ (comoving gauge), or $\zeta=0$ (zero curvature gauge), these surfaces have a well defined physical meaning in the following sense. Namely, choosing e.g. $\varphi=0$ completely fixes a gauge such that in that gauge $-2 \zeta$ can be associated with $W_{\zeta}$, and hence has a gauge invariant meaning. In that sense we can talk about a gauge invariant choice of hypersurfaces, $\Sigma_{W_{\zeta}}$ and $\Sigma_{W_{\varphi}}$ being just examples of two commonly used hypersurfaces. In this language, the question on which hypersurface one should perform calculations of non-Gaussianity becomes immaterial, as long as one knows on which hypersurface the observer measures, and how to relate the gauge invariant variables associated with the two hypersurfaces.
} 
The Einstein frame and the Jordan frame are related via a combined conformal transformation of the metric and a redefinition of the scalar field

$$
\begin{aligned}
g_{\mu \nu} & =\Omega^{2} g_{\mu \nu, J} \\
\left(\frac{d \Phi}{d \Phi_{J}}\right)^{2} & =\frac{1}{\Omega^{2}}+6 \frac{\Omega^{\prime 2}}{\Omega^{2}} \\
V(\Phi) & =\frac{1}{\Omega^{4}} V_{J}\left(\Phi_{J}\right),
\end{aligned}
$$

with

$$
\Omega^{2}=\Omega^{2}\left(\Phi_{J}\right)=F\left(\Phi_{J}\right) .
$$

Since these are just field redefinitions of the metric and scalar field, no physical information is expected to be lost in the frame transformation. This is what we refer to as physical equivalence of Jordan and Einstein frame. The physical equivalence is very useful, because it means we could obtain any results, such as the power spectrum, in the Jordan frame by transforming the well-known Einstein frame results using the above relations (6.2). Instead of dealing with the difficult nonminimal coupling, we merely have to deal with a modified potential.

Although the physical equivalence between Jordan and Einstein frame, in the sense described above, is well established at the classical level, it is not obvious that it also holds at the level of quantum fluctuations. In order to solve this issue, one could derive the tree-level action for cosmological perturbations in the Jordan frame. As a first step, we again slice up our space-time using the ADM metric (2.2) and insert perturbations similar to Eqs. (2.5),

$$
\begin{aligned}
g_{i j, J} & =a_{J}(t)^{2}\left(\delta_{i j}+h_{i j, J}(t, \vec{x})\right) \\
\Phi_{J} & =\phi_{J}(t)+\varphi_{J}(t, \vec{x}) \\
N_{J} & =\bar{N}_{J}(t)\left(1+n_{J}(t, \vec{x})\right) \\
N_{i, J} & =a_{J}(t) \bar{N}_{J}(t) n_{i, J}(t, \vec{x}) .
\end{aligned}
$$

The background Jordan frame action (6.1) then becomes

$$
S_{J}^{(0)}=\int d^{3} x d t \bar{N}_{J} a_{J}^{3}\left\{-3 H_{J}^{2} F-3 H_{J} \dot{F}+\frac{1}{2} \dot{\phi}_{J}^{2}-V_{J}\left(\phi_{J}\right)\right\}
$$

Here $H_{J}=\frac{\dot{a}_{J}}{a_{J}}=\frac{d a_{J} /\left(\bar{N}_{J} d t\right)}{a_{J}}$ and $\dot{\phi_{J}}=d \phi_{J} /\left(\bar{N}_{J} d t\right) .{ }^{9}$ Moreover, $F=F\left(\phi_{j}\right)$, so $F$ only depends on the background field $\phi_{J}$. The background equations in the Jordan frame are now obtained by varying the action with respect to $\bar{N}_{J}, a_{J}$ and $\phi_{J}$,

$$
\begin{aligned}
3 H_{J}^{2} F & =\frac{1}{2} \dot{\phi}_{J}^{2}+V_{J}\left(\phi_{J}\right)-3 H_{J} \dot{F} \\
2 \dot{H}_{J} F & =-\dot{\phi}_{J}^{2}+H_{J} \dot{F}-\ddot{F} \\
0 & =\ddot{\phi}_{J}+3 H_{J} \dot{\phi}_{J}-3\left(2 H_{J}^{2}+\dot{H}_{J}\right) F^{\prime}+V_{J}^{\prime}\left(\phi_{J}\right) .
\end{aligned}
$$

\footnotetext{
${ }^{9}$ A dotted derivative on a Jordan frame quantity implies that it is a derivative with respect to $\bar{N}_{J} d t$, whereas a dotted derivative on an Einstein frame quantity implies it is with respect to $\bar{N} d t$. We will use this consistently throughout this work.
} 
Using Eqs. (6.2) we can find the explicit relations between background quantities in the Jordan and Einstein frame,

$$
\begin{aligned}
\bar{N} & =\bar{\Omega} \bar{N}_{J} \\
a & =\bar{\Omega} a_{J} \\
H & =\frac{1}{\bar{\Omega}}\left(H_{J}+\frac{\dot{\bar{\Omega}}}{\bar{\Omega}}\right) \\
\dot{\phi} & =\frac{1}{\bar{\Omega}} \frac{d \phi}{d \phi_{J}} \dot{\phi}_{J} \equiv \frac{1}{\bar{\Omega}} \sqrt{\frac{1}{\bar{\Omega}^{2}}+6 \frac{\bar{\Omega}^{\prime 2}}{\bar{\Omega}^{2}}} \dot{\phi}_{J},
\end{aligned}
$$

where $\bar{\Omega}^{2} \equiv \Omega^{2}\left(\phi_{J}\right)=F\left(\phi_{J}\right)$. If we substitute these relations into the background action in the Einstein frame (2.1) or into the field equations (4.2), we immediately recover the background Jordan frame action (6.5) and its background field equations (6.6). This establishes the classical equivalence of Jordan and Einstein frame.

\subsection{Frame independence of second order action}

Next step is to find the second order action for cosmological perturbations in the Jordan frame by inserting (6.4) into (6.1). The nonminimal coupling between metric and scalar field is making this procedure much more complicated compared to the Einstein frame. It would be useful to establish also the frame equivalence at the level of perturbations, such that we can use the Einstein frame results and transform to the Jordan frame.

Before we proceed, we notice that the metric and field perturbations are not the same in Jordan and Einstein frame. The conformal factor $\Omega^{2}=\Omega^{2}\left(\Phi_{J}\right)$ can be perturbed to second order as

$$
\Omega\left(\Phi_{J}\right)=\bar{\Omega}+\Omega^{\prime} \varphi_{J}+\frac{1}{2} \Omega^{\prime \prime} \varphi_{J}^{2},
$$

where $\Omega^{\prime} \equiv d \Omega / d \phi_{J}$. Using this and the general relations between Jordan and Einstein frame (6.2), we can write second order relations for $\varphi$ and $\zeta$

$$
\begin{aligned}
\varphi & =\frac{d \phi}{d \phi_{J}} \varphi_{J}+\frac{1}{2} \frac{d^{2} \phi}{d \phi_{J}^{2}} \varphi_{J}^{2} \\
\zeta & =\zeta_{J}+\frac{\Omega^{\prime}}{\bar{\Omega}} \varphi_{J}+\frac{1}{2} \frac{\Omega^{\prime \prime}}{\bar{\Omega}} \varphi_{J}^{2}-\frac{1}{2}\left(\frac{\Omega^{\prime}}{\bar{\Omega}}\right)^{2} \varphi_{J}^{2} .
\end{aligned}
$$

Obviously the Jordan and Einstein frame perturbations of the scalar field and the scalar part of the metric are not the same. Consequently, neither $\varphi_{J}$ nor $\zeta_{J}$ are convenient variables to establish the quantum equivalence between the two frames, as these variables are inherently frame dependent. However, from the Einstein frame analysis we know that neither $\varphi$ nor $\zeta$ are physical perturbations, because they are gauge dependent. In the second order action for cosmological perturbations (4.6), we have seen that the only physical scalar perturbation is $w$, defined in (3.5). With the Jordan-Einstein frame relations for the background (6.7) and perturbations (6.9) at hand it is straightforward to show that at first order

$$
w=2 \frac{H}{\dot{\phi}} \varphi-2 \zeta=2 \frac{H_{J}}{\dot{\phi}_{J}} \varphi_{J}-2 \zeta_{J} \equiv w_{J} .
$$

The gauge invariant $w$ in Einstein frame coincides with the gauge invariant $w_{J}$ in the Jordan frame to first order. Thus the physical comoving curvature perturbation is not only gauge 
invariant, but frame independent as well. This frame independence to first order was first proven in Refs. $[18,19]$. Using this one can immediately write down the second order Jordan frame action via a frame transformation from the second order Einstein frame action (4.6), which was done in, for instance, Ref. [20]. By making use of the relation

$$
z^{2}=\frac{\dot{\phi}^{2}}{H^{2}}=\frac{1}{\overline{\Omega^{2}}} \frac{\dot{\phi}_{J}^{2}+6 \dot{\bar{\Omega}}^{2}}{\left(H_{J}+\frac{\dot{\bar{\Omega}}}{\bar{\Omega}}\right)^{2}} \equiv \frac{1}{\bar{\Omega}^{2}} z_{J}^{2},
$$

we can write

$$
S_{J}^{(2)}\left(w_{J}\right)=\int d^{3} x d t \bar{N}_{J} a_{J}^{3} \frac{1}{4} z_{J}^{2}\left\{\frac{1}{2} \dot{w}_{J}^{2}-\frac{1}{2}\left(\frac{\partial_{i} w_{J}}{a_{J}}\right)^{2}\right\} .
$$

Of course the second order action (6.12) is also obtained by starting with the original Jordan frame action (6.1) and doing the same steps as in the Einstein frame derivation. That is, use the ADM decomposition, solve for the constraint fields to first order, and write the action for physical perturbations. In Ref. [21] this was done without solving for the constraint fields, but by decoupling the constraint degrees of freedom from the dynamical degrees of freedom. The result for the scalar dynamical degree of freedom agrees with (6.12). This establishes the equivalence of frames at the level of quadratic perturbations.

Note that when the action for perturbations is written in terms of gauge invariant and frame independent perturbations, there is no notion of separate frames. Although the Jordan frame and Einstein frame actions (6.1) and (2.1) look originally quite different (nonminimal or minimal coupling), at the level of perturbations the actions have a unique form. Therefore it does not make sense to talk about a preferred frame to describe perturbations. One can choose to start with an action for a certain nonminimally coupled scalar field, or for another minimally coupled scalar field (related to the first via the relation (6.2)) in a modified potential, and both give equivalent results. Of course, in the end one wants to express the results (such as $n$-point functions) in terms of variables in the defining frame.

As an example, in Higgs inflation the defining frame is the Jordan frame, where the scalar field $\Phi_{J}$ is identified with the Higgs field, the potential $V_{J}$ is the Higgs potential and the field is coupled to the scalar curvature via $F\left(\Phi_{J}\right)=1+\xi \Phi_{J}^{2}$. One can directly compute perturbations in the Jordan frame, resulting in the manifestly gauge invariant quadratic action (6.12), but the derivation is difficult because of the nonminimal coupling. It is easier to transform to the Einstein frame first, and then use the standard result (4.6). Of course the scalar field in the Einstein frame is not the Higgs field, but is related to the Higgs field via a field redefinition, just like the Einstein frame metric. After finding the standard Einstein frame results one can transform back to the Jordan frame (the defining frame) in order to obtain (6.12).

\subsection{Frame independence of cubic action}

In the previous section it was shown that the comoving curvature perturbation in the Einstein frame coincides with the same perturbation in the Jordan frame at first order. At second order however $w$ and $w_{J}$ are related as

$$
w=w_{J}+\left[\left(\frac{H_{J}}{\dot{\phi}_{J}}+\frac{\Omega^{\prime}}{\bar{\Omega}}\right) \frac{d^{2} \phi / d \phi_{J}^{2}}{d \phi / d \phi_{J}}-\frac{\Omega^{\prime \prime}}{\bar{\Omega}}+\left(\frac{\Omega^{\prime}}{\bar{\Omega}}\right)^{2}\right] \varphi_{J}^{2}
$$

Thus the first order gauge invariant variable $w$ is not a convenient variable for comparing Jordan frame results with Einstein frame results at third order, since the variable itself is frame 
dependent. Motivated by the results in the previous section, we could check if a second order gauge invariant variable is frame independent, in the sense that it has exactly the same form in the Jordan and Einstein frames. Indeed, it can be shown that the curvature perturbation on uniform field hypersurfaces, $W_{\zeta}$ from Eq. (3.9), coincides with the same gauge invariant variable in the Jordan frame,

$$
\begin{aligned}
W_{\zeta} & =w-\left[\left(\frac{\ddot{\phi}}{H \dot{\phi}}-\frac{\dot{H}}{H^{2}}\right) \frac{H^{2}}{\dot{\phi}^{2}} \varphi^{2}+\frac{1}{\dot{\phi}} \dot{w} \varphi\right] \\
& =w_{J}-\left[\left(\frac{\ddot{\phi}_{J}}{H_{J} \dot{\phi}_{J}}-\frac{\dot{H}_{J}}{H_{J}^{2}}\right) \frac{H_{J}^{2}}{\dot{\phi}_{J}^{2}} \varphi_{J}^{2}+\frac{1}{\dot{\phi}_{J}} \dot{w}_{J} \varphi_{J}\right] \\
& \equiv W_{\zeta, J}
\end{aligned}
$$

where we did not write the terms with vectors, tensors or spatial derivatives. The frame independence of $W_{\zeta}$ was shown explicitly in [22], but other, more general, proofs exist as well [23-25]. This means that we can directly find the third order action in the Jordan frame from the third order Einstein frame action expressed in terms of $W_{\zeta}$, which can be used, for example, to find $f_{\mathrm{NL}}$ for a nonminimally coupled theory $[22,23,26]$. We only have to replace the background Einstein frame quantities by corresponding Jordan frame quantities using (6.7). Thus from (4.24) we straightforwardly find

$$
\begin{aligned}
\tilde{S}_{\mathrm{GI}}^{(3)}\left(W_{\zeta}\right)= & \int d^{3} x d t \frac{\bar{N}_{J} a_{J}^{3}}{8}\left\{\frac{1}{2} z_{J}^{2} W_{\zeta, J}\left(\frac{\partial_{i} W_{\zeta, J}}{a_{J}}\right)^{2}-\frac{3}{2} z_{J}^{2} W_{\zeta, J} \dot{W}_{\zeta, J}^{2}+\frac{1}{2} \frac{\bar{\Omega} z_{J}^{2}}{H_{J}+\frac{\overline{\bar{\Omega}}}{\bar{\Omega}}} \dot{W}_{\zeta, J}^{3}\right. \\
& \left.-\frac{1}{2}\left(3 W_{\zeta, J}-\frac{\bar{\Omega} \dot{W}_{\zeta, J}}{H_{J}+\frac{\dot{\bar{\Omega}}}{\Omega}}\right)\left[\frac{\partial_{i} \partial_{j} \psi_{J}}{a_{J}^{2}} \frac{\partial_{i} \partial_{j} \psi_{J}}{a_{J}^{2}}-\left(\frac{\nabla^{2} \psi_{J}}{a_{J}^{2}}\right)^{2}\right]+2 \frac{\partial_{i} \psi_{J}}{a_{J}} \frac{\partial_{i} W_{\zeta, J}}{a_{J}} \frac{\nabla^{2} \psi_{J}}{a_{J}^{2}}\right\}
\end{aligned}
$$

where

$$
\frac{\nabla^{2} \psi_{J}}{a_{J}^{2}}=-\frac{\nabla^{2}}{a_{J}^{2}} \frac{\bar{\Omega} W_{\zeta, J}}{H_{J}+\frac{\dot{\bar{\Omega}}}{\bar{\Omega}}}+\frac{1}{2} z_{J}^{2} \dot{W}_{\zeta_{J}} .
$$

The 3-point function in for a non-minimally coupled theory is found in a similar way. Thus, one first transforms transforms to the Einstein frame where one can use previously computed results for the 3-point function for $W_{\zeta}$, and this result can be expressed in Jordan frame quantities by going back to the Jordan frame.

Of course we can also transform the partially integrated Einstein frame action for $W_{\zeta}(5.12)$, that shows the separation between different orders in slow-roll, to the Jordan frame. After the frame transformations we find

$$
\begin{aligned}
\tilde{S}_{\mathrm{GI}, J}^{(3)}= & \int d^{3} x d t \frac{\bar{N}_{J} a_{J}^{3}}{8}\left\{\frac{1}{2} \frac{z_{J}^{4}}{\bar{\Omega}^{2}}\left[-\frac{1}{2} \dot{W}_{\zeta, J}^{2} W_{\zeta, J}-\frac{1}{2}\left(\frac{\partial_{i} W_{\zeta, J}}{a_{J}}\right)^{2} W_{\zeta, J}+\dot{W}_{\zeta, J}\left(\frac{\partial_{i}}{\nabla^{2}} \dot{W}_{\zeta, J}\right) \partial_{i} W_{\zeta, J}\right]\right. \\
& +\frac{1}{16} \frac{z_{J}^{6}}{\bar{\Omega}^{4}}\left[\dot{W}_{\zeta, J}^{2} W_{\zeta, J}-\left(\frac{\partial_{i} \partial_{j}}{\nabla^{2}} \dot{W}_{\zeta, J}\right)\left(\frac{\partial_{i} \partial_{j}}{\nabla^{2}} \dot{W}_{\zeta, J}\right) W_{\zeta, J}\right]-\frac{1}{2} z_{J}^{2} \dot{W}_{\zeta, J} W_{\zeta, J}^{2}\left[\frac{\bar{\Omega}\left(z_{J} / \bar{\Omega}\right)^{\cdot}}{z_{J}\left(H_{J}+\frac{\dot{\bar{\Omega}}}{\Omega}\right)}\right] \\
& \left.+\frac{1}{a_{J}^{3}} \frac{\delta S_{J}^{(2)}\left(w_{J}\right)}{\delta w_{J}}\left[\frac{\bar{\Omega}}{4 z_{J}\left(H_{J}+\frac{\dot{\bar{\Omega}}}{\Omega}\right)}\left(z_{J} W_{\zeta, J}^{2} / \bar{\Omega}\right)\right]\right\} .
\end{aligned}
$$


As explained in Sec. 5, the boundary terms are accounted for by performing a field redefinition to a new gauge invariant variable

$$
\tilde{W}_{\varphi, J}=W_{\zeta, J}+\frac{\bar{\Omega}}{4 z_{J}\left(H_{J}+\frac{\dot{\bar{\Omega}}}{\Omega}\right)}\left(z_{J} W_{\zeta, J}^{2} / \bar{\Omega}\right)^{.} .
$$

The 3-point function for $W_{\zeta, J}$ is then calculated from the 3-point function for $\tilde{W}_{\varphi, J}$ plus disconnected parts in that variable

$$
\begin{aligned}
& \left\langle W_{\zeta, J}\left(\vec{x}_{1}\right) W_{\zeta, J}\left(\vec{x}_{2}\right) W_{\zeta, J}\left(\vec{x}_{3}\right)\right\rangle=\left\langle\tilde{W}_{\varphi, J}\left(\vec{x}_{1}\right) \tilde{W}_{\varphi, J}\left(\vec{x}_{2}\right) \tilde{W}_{\varphi, J}\left(\vec{x}_{3}\right)\right\rangle \\
& +\frac{1}{2} \frac{\dot{z_{J}}-\frac{\dot{\bar{\Omega}}}{\Omega}}{z_{J}\left(H_{J}+\frac{\dot{\bar{\Omega}}}{\Omega}\right)}\left(\left\langle\tilde{W}_{\varphi, J}\left(\vec{x}_{1}\right) \tilde{W}_{\varphi, J}\left(\vec{x}_{2}\right)\right\rangle\left\langle\tilde{W}_{\varphi, J}\left(\vec{x}_{1}\right) \tilde{W}_{\varphi, J}\left(\vec{x}_{3}\right)\right\rangle+\mathrm{sym}\right)+\ldots,
\end{aligned}
$$

where $z_{J}$ is defined in Eq. (6.11) and terms of higher order in slow-roll have been neglected. The variable $\tilde{W}_{\varphi, J}$ is of course the frame transformed $W_{\varphi}$, which is seen most easily after transforming both sides of the non-linear relation between gauge invariant variables (3.10). $\tilde{W}_{\varphi, J}$ is however not directly related to the field perturbation on uniform curvature hypersurfaces in the Jordan frame, since it does not reduce to $2 \frac{H_{J}}{\dot{\phi}_{J}} \varphi_{J}$ in the gauge $\zeta_{J}=0$. In fact, the variable

$$
W_{\varphi} \equiv w_{J}-\left[\left(\frac{\ddot{\phi}_{J}}{H_{J} \dot{\phi}_{J}}-\frac{\dot{H}_{J}}{H_{J}^{2}}\right)\left(\zeta_{J}^{2}+w_{J} \zeta_{J}\right)+\frac{1}{H} \dot{w} \zeta_{J}\right] \text {, }
$$

is precisely the gauge invariant variable in the Jordan frame that does this. The question is how to compute the 3-point function for the field perturbation on uniform curvature hypersurfaces in the Jordan frame: what is the action for $W_{\varphi, J}$ ? It can be shown that $W_{\varphi, J}$ is non-linearly related to $\tilde{W}_{\varphi, J}$ as

$$
\tilde{W}_{\varphi, J}=W_{\varphi, J}^{2}+\frac{1}{4}\left[\frac{\dot{z_{J}}-\frac{\dot{\bar{\Omega}}}{\bar{\Omega}}}{z_{J}\left(H_{J}+\frac{\dot{\bar{\Omega}}}{\Omega}\right)}-\frac{1}{\dot{\phi}_{J}}\left(\frac{\dot{\phi}_{J}}{H_{J}}\right)\right] W_{\varphi, J}^{2},
$$

where terms higher order in slow-roll have been neglected. In the previous chapter 5 we have shown that the cubic actions for non-linearly related variables differ only by boundary terms, which can give disconnected contributions to the bispectrum. Thus the bispectrum for the field perturbation on uniform curvature hypersurfaces in the Jordan frame contains a connected part coming from the first lines of the action (6.17), plus a disconnected part from the non-linear relation (6.21). Alternatively, one could use the direct relation

$$
W_{\varphi, J}=W_{\zeta, J}-\frac{1}{4} \frac{1}{\dot{\phi}_{J}}\left(\frac{\dot{\phi}_{J}}{H_{J}}\right) W_{\zeta, J}^{2},
$$

by replacing all quantities by Jordan frame quantities in (3.10). Then one finds the connected part of the bispectrum for $W_{\varphi, J}$ from (6.15), and disconnected pieces from (6.22).

Now some words about the special situation when $\varphi_{J}=0$. In that case the conformal factor $\Omega^{2}$ only has a background value and $W_{\zeta}(\varphi=0)=\zeta_{J}=\zeta$. Thus the cubic terms in $\zeta_{J}$ not only directly provide the gauge invariant vertices, but can also be transformed to Einstein frame vertices, and vice versa. The third order Jordan frame action for $\zeta_{J}$ was derived in [27], and 
it was shown that it can be found from the Einstein frame action [1-3] in Ref. [28]. Moreover, one could imagine that at higher order in perturbations one can construct a gauge invariant variable which reduces to $\zeta_{J}$ in the gauge $\varphi_{J}=0$, just as we did before for $W_{\zeta}$ to second order. When $\varphi_{J}=0$ it now becomes almost trivial to show that the curvature perturbation is invariant under frame transformations. Thus, the curvature perturbation on uniform field hypersurfaces is frame independent to all orders [25].

Finally a remark about previous results found in Higgs inflation. Often computations are done in both frames, examples being quantum corrections of the Higgs potential [29-33], or the computations of the cut-off scale for which the theory becomes nonperturbative [34-39]. The Einstein frame results are then compared to direct Jordan frame computations by transforming them to the Jordan frame. More often than not, transformed Einstein frame results do not exactly agree with what is found in the Jordan frame. A recent example is a calculation of the field dependent cut-off in [39], which appears different in one frame or the other. However, the result of this section is that the Jordan frame action can be found directly from the Einstein frame via a field transformation. The most clear way to see this is that everything can be expressed in frame independent variables. Thus the cut-off scale should be the same whether you compute it directly in the Jordan frame, or via transformed Einstein frame results. The reason for the confusion and difference between results obtained in different frames in the references above is due to a non-covariant formalism, where the variables become frame dependent. For example, in Einstein frame computations often the nonperturbative cut-off scale is found from expanding the non-polynomial potential in powers of $\varphi$ and neglecting metric fluctuations. $\varphi$ is then a frame dependent variable. There is no confusion when using frame independent variables: quantum corrections or cut-off's computed directly in the Jordan frame are exactly the same when first computed in the Einstein frame and transformed back to the Jordan frame. The cut-off can therefore in principle be computed directly from (6.15), and from higher order generalisation of that action.

\section{Summary and conclusion}

In this work we have focused on scalar perturbations of a scalar field and the metric around an expanding background. These perturbations are generally gauge dependent. It is possible to construct gauge invariant variables by taking certain combinations of these perturbations. At second order in coordinate transformations there are in principle infinitely many gauge invariant variables, two specific examples being the comoving curvature perturbation and the field perturbation on uniform curvature hypersurfaces. These variables are related in a non-linear way.

We have outlined the procedure for finding the gauge invariant actions for these variables at third order, and we have shown explicitly the gauge invariant cubic actions. Next we have demonstrated that, due to the non-linear relation between these variables, the cubic actions appear different, but actually their bulk actions are the same. They differ by boundary terms, which generically give disconnected contributions to the bispectrum.

This brings us to the aspect of uniqueness. Once you pick a certain initial hypersurface, or equivalently choose a specific gauge invariant variable, for example the comoving curvature perturbation in a Gaussian state, then there is a unique action for this variable that evolves the initial (Gaussian) state and creates non-Gaussianity through the evolution. If the final hypersurface is different then the initial, boundary terms must be taken into account which appear when switching to a different gauge invariant variable associated with the final hypersurface. 
We also commented on initial non-Gaussianity: although for one gauge invariant variable the initial state is Gaussian, it is generically non-Gaussian for another variable. Some of the final non-Gaussianity for a certain variable therefore originates from some initial non-Gaussianity.

Finally we discussed different conformally related frames. It was shown that the cubic action for gauge invariant perturbations in the Jordan frame can be obtained directly from the Einstein frame action. The trick is to identify the variable that has exactly the same form in either frame. The comoving curvature perturbation is such a frame independent cosmological perturbation. Thus the bispectrum for the comoving curvature perturbation can be found from the Einstein frame bispectrum by transforming the result to the Jordan frame. The bispectrum for another gauge invariant variable in the Jordan frame, such as the field perturbation on uniform curvature hypersurfaces, is then found from the non-linear relation between this variable and the comoving curvature perturbation.

Frame independent cosmological perturbations can be a very useful tool in calculating quantum corrections for non-minimally coupled theories. An example is Higgs inflation, where often different results are found depending on which frame one uses for the computations due to non-covariance of the perturbed actions. These differences can be attributed to both frame dependence and gauge dependence of the formalism used. The issue is resolved when using frame independent, gauge invariant variables, since then the Jordan and Einstein frame actions have exactly the same form.

The techniques used here can be quite straightforwardly generalised to compute higher order (quartic, etc.) gauge invariant tree level actions for scalar cosmological perturbations (needless to say, the choice of gauge invariant variables at cubic and higher orders is much richer), and it is the subject of future study. Higher order gauge invariant actions are the necessary tool for gauge invariant calculations of quantum corrections to cosmological observables, the simplest one being the one loop correction to the equal time two point function for scalar cosmological perturbations. This work represents a step towards establishing a fully covariant framework for calculating quantum field theoretic correlators in curved space time backgrounds. Cosmology is a field where such correlators may in fact be observable. The problem of observables in cosmology is one of the most important unsolved problems in theoretical cosmology[40].

\section{Acknowledgements}

We would like to thank Gerasimos Rigopoulos for useful discussions and comments. This research was supported by the Dutch Foundation for 'Fundamenteel Onderzoek der Materie' (FOM) under the program "Theoretical particle physics in the era of the LHC", program number FP 104.

\section{References}

[1] J. M. Maldacena, Non-Gaussian features of primordial fluctuations in single field inflationary models, JHEP 05 (2003) 013, [astro-ph/0210603].

[2] D. Seery and J. E. Lidsey, Primordial non-Gaussianities in single field inflation, JCAP 0506 (2005) 003, [astro-ph/0503692].

[3] X. Chen, M.-x. Huang, S. Kachru, and G. Shiu, Observational signatures and non-Gaussianities of general single field inflation, JCAP 0701 (2007) 002, [hep-th/0605045].

[4] J. M. Bardeen, Gauge Invariant Cosmological Perturbations, Phys. Rev. D22 (1980) 1882-1905.

[5] R. L. Arnowitt, S. Deser, and C. W. Misner, The dynamics of general relativity, gr-qc/0405109. 
[6] T. Prokopec and G. Rigopoulos, Path Integral for Inflationary Perturbations, Phys. Rev. D82 (2010) 023529, [arXiv: 1004.0882].

[7] M. Bruni, S. Matarrese, S. Mollerach, and S. Sonego, Perturbations of spacetime: Gauge transformations and gauge invariance at second order and beyond, Class. Quant. Grav. 14 (1997) 2585-2606, [gr-qc/9609040].

[8] K. A. Malik and D. Wands, Cosmological perturbations, Phys. Rept. 475 (2009) 1-51, [arXiv:0809.4944].

[9] H. Noh and J.-c. Hwang, Second-order perturbations of the Friedmann world model, Phys. Rev. D69 (2004) 104011.

[10] G. Rigopoulos, Gauge invariance and non-Gaussianity in Inflation, Phys.Rev. D84 (2011) 021301, [arXiv: 1104.0292].

[11] K. A. Malik and D. Wands, Evolution of second-order cosmological perturbations, Class.Quant.Grav. 21 (2004) L65-L72, [astro-ph/0307055].

[12] V. F. Mukhanov, H. A. Feldman, and R. H. Brandenberger, Theory of cosmological perturbations. Part 1. Classical perturbations. Part 2. Quantum theory of perturbations. Part 3. Extensions, Phys. Rept. 215 (1992) 203-333.

[13] S. Weinberg, Quantum contributions to cosmological correlations, Phys.Rev. D72 (2005) 043514, [hep-th/0506236].

[14] F. Arroja and T. Tanaka, A note on the role of the boundary terms for the non-Gaussianity in general k-inflation, JCAP 1105 (2011) 005, [arXiv:1103.1102].

[15] C. Burrage, R. H. Ribeiro, and D. Seery, Large slow-roll corrections to the bispectrum of noncanonical inflation, JCAP 1107 (2011) 032, [arXiv:1103.4126].

[16] D. S. Salopek, J. R. Bond, and J. M. Bardeen, Designing Density Fluctuation Spectra in Inflation, Phys. Rev. D40 (1989) 1753.

[17] F. L. Bezrukov and M. Shaposhnikov, The Standard Model Higgs boson as the inflaton, Phys. Lett. B659 (2008) 703-706, [arXiv:0710.3755].

[18] N. Makino and M. Sasaki, The Density perturbation in the chaotic inflation with nonminimal coupling, Prog. Theor. Phys. 86 (1991) 103-118.

[19] R. Fakir, S. Habib, and W. Unruh, Cosmological density perturbations with modified gravity, Astrophys. J. 394 (1992) 396.

[20] J.-c. Hwang, Cosmological perturbations in generalized gravity theories: Conformal transformation, Class. Quant. Grav. 14 (1997) 1981-1991, [gr-qc/9605024].

[21] J. Weenink and T. Prokopec, Gauge invariant cosmological perturbations for the nonminimally coupled inflaton field, Phys.Rev. D82 (2010) 123510, [arXiv:1007.2133].

[22] N. Sugiyama and T. Futamase, Non-Gaussianity generated in the inflationary scenario with nonminimally coupled inflaton field, Phys.Rev. D81 (2010) 023504.

[23] S. Koh, Non-gaussianity in nonminimally coupled scalar field theory, J.Korean Phys.Soc. 49 (2006) S787-S790, [astro-ph/0510030].

[24] T. Chiba and M. Yamaguchi, Extended Slow-Roll Conditions and Rapid-Roll Conditions, JCAP 0810 (2008) 021, [arXiv:0807.4965].

[25] J.-O. Gong, J.-c. Hwang, W.-I. Park, M. Sasaki, and Y.-S. Song, Conformal invariance of curvature perturbation, JCAP 1109 (2011) 023, [arXiv:1107.1840].

[26] S. Koh, S. P. Kim, and D. J. Song, Nonlinear evolutions and non-Gaussianity in generalized gravity, Phys.Rev. D71 (2005) 123511, [astro-ph/0501401]. 
[27] T. Qiu and K.-C. Yang, Non-Gaussianities of Single Field Inflation with Non-minimal Coupling, Phys.Rev. D83 (2011) 084022, [arXiv:1012.1697].

[28] T. Kubota, N. Misumi, W. Naylor, and N. Okuda, The Conformal Transformation in General Single Field Inflation with Non-Minimal Coupling, JCAP 1202 (2012) 034, [arXiv:1112.5233].

[29] F. L. Bezrukov, A. Magnin, and M. Shaposhnikov, Standard Model Higgs boson mass from inflation, Phys. Lett. B675 (2009) 88-92, [arXiv:0812.4950].

[30] F. Bezrukov and M. Shaposhnikov, Standard Model Higgs boson mass from inflation: two loop analysis, JHEP 07 (2009) 089, [arXiv:0904.1537].

[31] A. O. Barvinsky, A. Y. Kamenshchik, and A. A. Starobinsky, Inflation scenario via the Standard Model Higgs boson and LHC, JCAP 0811 (2008) 021, [arXiv:0809.2104].

[32] A. O. Barvinsky, A. Y. Kamenshchik, C. Kiefer, A. A. Starobinsky, and C. Steinwachs, Asymptotic freedom in inflationary cosmology with a non- minimally coupled Higgs field, JCAP 0912 (2009) 003, [arXiv:0904.1698].

[33] A. De Simone, M. P. Hertzberg, and F. Wilczek, Running Inflation in the Standard Model, Phys. Lett. B678 (2009) 1-8, [arXiv:0812.4946].

[34] R. N. Lerner and J. McDonald, Higgs Inflation and Naturalness, JCAP 1004 (2010) 015, [arXiv: 0912.5463].

[35] J. L. F. Barbon and J. R. Espinosa, On the Naturalness of Higgs Inflation, Phys. Rev. D79 (2009) 081302, [arXiv:0903.0355].

[36] C. P. Burgess, H. M. Lee, and M. Trott, Comment on Higgs Inflation and Naturalness, JHEP 07 (2010) 007, [arXiv: 1002.2730].

[37] M. P. Hertzberg, On Inflation with Non-minimal Coupling, JHEP 1011 (2010) 023, [arXiv: 1002.2995].

[38] F. Bezrukov, A. Magnin, M. Shaposhnikov, and S. Sibiryakov, Higgs inflation: consistency and generalisations, JHEP 1101 (2011) 016.

[39] F. Bezrukov, D. Gorbunov, and M. Shaposhnikov, Late and early time phenomenology of Higgs-dependent cutoff, JCAP 1110 (2011) 001, [arXiv:1106. 5019].

[40] R. P. Woodard, Talk at the CERN 2011 workshop on "Quantum Gravity: from UV to IR". 